\title{
Construction of Markov processes and associated multiplicative functionals from given harmonic measures
}

\author{
C.T. Shih \\ Mathematics Department, University of Michigan, Ann Arbor, MI 48109, USA
}

Received: 12 December 1992 / In revised form: 21 September 1993

\begin{abstract}
Summary. Let $E$ be a noncompact locally compact second countable Hausdorff space. We consider the question when, given a family of finite nonzero measures on $E$ that behave like harmonic measures associated with all relatively compact open sets in $E$ (i.e. that satisfy a certain consistency condition), one can construct a Markov process on $E$ and a multiplicative functional with values in $[0, \infty)$ such that the hitting distributions of the process "inflated" by the multiplicative functional yield the given harmonic measures. We achieve this construction under weak continuity and local transience conditions on these measures that are natural in the theory of Markov processes, and a mild growth restriction on them. In particular, if the space $E$ equipped with the measures satisfies the conditions of a harmonic space, such a Markov process and associated multiplicative functional exist. The result extends in a new direction the work of many authors, in probability and in axiomatic potential theory, on constructing Markov processes from given hitting distributions (i.e. from harmonic measures that have total mass no more than 1).
\end{abstract}

Mathematics Subject Classification (1991): 60J40, 60J45, 31D05

\section{Introduction}

Let $E$ be a locally compact second countable Hausdorff space. Let $\left\{E_{n}\right\}$ be a (countable) open covering of $E$ where each $E_{n}$ is relatively compact and has a nonempty complement. Denote

$$
\mathscr{U}=\left\{U: U \text { is open, } U \subset E_{n} \text { for some } n\right\}, \quad \mathscr{D}=\{D: D=E-U \text { for sorne } U \in \mathscr{U}\} .
$$

Consider a family of nonzero finite measures $Q_{D}(x, \cdot)$ on $E$, where $D \in \mathscr{D}, x \in E$, such that each $Q_{D}(x, \cdot)$ is concentrated on $D$, if $x \in D$ it is the point mass at $x$, and $Q_{D}(\cdot, B)$ is Borel measurable (or more generally nearly-Borel measurable - see Sect. 1) if $B$ is Borel. Assume the family satisfies the consistency condition that, if $F \subset D, Q_{F}(x, \cdot)=\int Q_{D}(x, d y) Q_{F}(y, \cdot)$. The $Q_{D}(x, \cdot)$ are then called harmonic measures, (and one may call the space $E$ equipped with such a family $Q_{D}(x, \cdot)$ a general harmonic or balayage space). It is natural to ask that under what 
(additional) conditions on the $Q_{D}(x, \cdot)$ one can construct a Markov process (more precisely, a right process) $Y=\left(Y_{t}, P^{x}, \zeta\right)$ on $E$, $\zeta$ the lifetime, and a multiplicative functional $\left(M_{t}\right)$ in $Y$ with values in $[0, \infty)$ such that for all $x, D \in \mathscr{D}$ and bounded Borel $f$ on $E$

$$
\begin{aligned}
\int Q_{D}(x, d y) f(y) & =P^{x}\left[f\left(Y\left(T_{D}\right)\right) M\left(T_{D}\right) ; T_{D}<\zeta\right] \\
& :=\int_{\left[T_{D}<\zeta\right]} f\left(Y\left(T_{D}\right)\right) M\left(T_{D}\right) d P^{x}
\end{aligned}
$$

where $T_{D}=\inf \left\{t \geqq 0: Y_{t} \in D\right\}$, the (first) hitting time of $D$.

When the $Q_{D}(x, \cdot)$ are subprobability measures, the multiplicative functional is not needed, and the problem is that of constructing a Markov process $Y$ with the $Q_{D}(x, \cdot)$ as its hitting distributions $P^{x}\left[Y\left(T_{D}\right) \in \cdot, T_{D}<\zeta\right]$. Since 1963 this latter problem has been studied in a number of papers, by P.A. Meyer, F. Knight and S. Orey, D.A. Dawson, N. Boboc and C. Constantinescu and A. Cornea, W. Hansen, C.T. Shih, J.C. Taylor, J. Bliedtner and W. Hansen, J.B. Gravereux and J. Jacod; see references in [9]. Different constructions under different sets of conditions were introduced in these papers, some of which use mostly potential theoretical methods. All of these papers assume stronger continuity conditions than [7], which constructs all transient Hunt processes (up to a time change) and whose result is needed in this article. (We note that, using the theorem in [8], the construction in [7] easily extends to the recurrent Hunt processes as well, and the result is stated as Theorem 3 in [9]; [9] also corrected a gap in [7] in the proof of convergence of the time scale when holding points exist.)

In the present article the measures $Q_{D}(x, \cdot)$ may have total mass greater than 1 . We assume the following natural conditions on the $Q_{D}(x, \cdot)$ : local transience, intrinsic right continuity, and quasi-left-continuity, (see Sect. 1 for their meaning). Furthermore assume the following growth condition: if $F \subset D^{\circ}$ (interior of $D$ ) then for any $x$

$$
\int Q_{D}(x, d y) q_{F}(y) \log q_{F}(y)<\infty
$$

where $q_{F}(y)=Q_{F}(y, E)$. (Note that $\int Q_{D}(x, d y) q_{F}(y)=q_{F}(x)<\infty$ by the consistency condition; so (0.2) is not a severe restriction.) The following result is proved. Fix for each $n$ an open set $U_{n}$ with closure $\bar{U}_{n} \subset E_{n}$ and denote $\mathscr{D}^{\prime}=\{D \in \mathscr{D}$ : $E-D \subset U_{n}$ for some $\left.n\right\}$; note that if there is a subsequence of $E_{n}$ increasing to $E$, the $U_{n}$ can be chosen so that $\mathscr{D}^{\prime}=\mathscr{D}$. Then there exists a right process $Y$ on $E$, which is actually a standard process because of the quasi-left-continuity, and a multiplicative functional $\left(M_{t}\right)$ such that $(0.1)$ holds for all $x, D \in \mathscr{D}^{\prime}$ and bounded Borel $f$ on $E$.

To our knowledge this result is new even in the context of the theory of harmonic spaces (see e.g. Constantinescu and Cornea [3]) and the more recent theory of balayage spaces (see Bliedtner and Hansen [1]), where the continuity conditions assumed are substantially stronger than the one assumed in this article, which consists of the intrinsic right continuity (a necessary condition) and the quasi-left-continuity. In this connection, however, it should be noted that our method is entirely probabilistic.

Incidentally, the continuity condition assumed in the theory of harmonic spaces (or in that of balayage spaces) implies that for all bounded Borel $f$ the function $y \rightarrow \int Q_{F}(y, d z) f(z)$ is continuous on $E-F$; therefore $q_{F}(y)$ is contin- 
uous on $E-F$ and so locally bounded there, and consequently, since in a harmonic space each $Q_{D}(x, \cdot)$ is concentrated on the boundary of $D$ if $x \notin D$, the growth conditions $(0.2)$ is always satisfied in such a space.

The results are stated in Sect. 1. In Sect. 2 "local processes" are constructed based on the result in [7]. The heart of this work, however, is in Sects. 3 and 4 , where "transformation (multiplicative) functionals" are constructed to connect the local processes, from which a consistent family of local processes and associated local multiplicative functionals are obtained, and finally a global process and associated multiplicative functional are defined by piecing together the local ones (with time changes). The reader not familiar with [7], but with one of the constructions (of a Markov process from hitting distributions) referred to above, may replace (some of) the hypotheses in Sect. 1 by his/her familiar ones, omit reading Sect. 2 except the definitions of local processes and their associated local multiplicative functionals (see Theorem 2.1), and proceed to read Sects. 3 and 4 .

\section{Statement of results}

As in the introduction $E$ is a locally compact second countable Hausdorff space. Let $E_{\Delta}=E \cup\{\Delta\}$ be its one-point compactification. Let $\mathscr{E}_{\Delta}$ (resp. $\mathscr{E}_{A}^{*}$ ) denote the $\sigma$-algebra of Borel (resp. universally measurable) sets in $E_{\Delta} . f \in b \mathscr{E}_{\Delta}$ (resp. $f \in \mathscr{E}_{A}^{+}$), e.g., means $f$ is a bounded (resp. positive) real $\mathscr{E}_{A}$-measurable function. All real functions $f$ on $E_{\Delta}$ will satisfy $f(\Delta)=0$ as a convention.

Let $\left\{E_{n}, n \geqq 1\right\}$ be an open covering of $E$ where each $E_{n}$ has (compact) closure $\bar{E}_{n} \subset E$, and $E-E_{n}$ is nonempty. (Again) let

$$
\begin{aligned}
& \mathscr{U}=\left\{U: U \text { is open, } U \subset E_{n} \text { for some } n\right\} \\
& \mathscr{D}=\left\{D: D=U^{c}=E_{\Delta}-U \text { for some } U \in \mathscr{U}\right\} .
\end{aligned}
$$

Consider a family $\left\{Q_{D}(x, \cdot): D \in \mathscr{D}, x \in D\right\}$ of (positive) measures on $E_{\Delta}$ that satisfies the following hypotheses (Q1) through (Q7).

(Q1) $0<q_{D}(x):=Q_{D}\left(x, E_{A}\right)<\infty ; Q_{D}(x, \cdot)$ is concentrated on $D ; Q_{D}(x, \cdot)$ is the point mass $\varepsilon_{x}$ if $x \in D ; Q_{D}(x,\{\Delta\})=0$ if $x \neq \Delta ; Q_{D}(\cdot, B) \in \mathscr{E}_{A}^{*}$ if $B \in \mathscr{E}_{A}$.

(Q2) (Consistency) If $F \subset D, Q_{F}(x, \cdot)=\int Q_{D}(x, d y) Q_{F}(y, \cdot)$, i.e. $Q_{D}\left(x, Q_{F} f\right)$ $=Q_{F}(x, f)$ for all $f \in b \mathscr{E}_{\Delta}^{*}$ where $Q_{D} f(y)=Q_{D}(y, f):=\int Q_{D}(y, d z) f(z)$.

To state the next hypothesis, we need to define the nearly Borel sets (relative to the family $\left.Q_{D}(x, \cdot)\right)$. A set $A \subset E_{A}$ is nearly Borel if for every $n$ and finite measure $\mu$ on $E_{A}$ there exist Borel sets $B_{1}, B_{2}$ with $B_{1} \subset A \cap E_{n} \subset B_{2} \subset E_{n}$ such that for all compact $C \subset B_{2}-B_{1}$ we have

$$
\int \mu(d x) Q_{E_{n}^{c} \cup c}(x, C)=0 .
$$

It will be seen that the family $\mathscr{E}_{\Delta}^{n}$ of nearly Borel sets is a $\sigma$-algebra. It is easy to see that $\mathscr{E}_{\Delta} \subset \mathscr{E}_{\Delta}^{n} \subset \mathscr{E}_{\Delta}^{*}$.

(Q3) (Nearly-Borel measurability) $Q_{D}(\cdot, B) \in \mathscr{E}_{\Delta}^{n}$ if $B \in \mathscr{E}_{\Delta}$.

(Q4) (Local transience) If $F \subset D$ and $x \notin D$, then for some compact neighborhood $C$ of $x$ with $C \subset F^{c}$

$$
\int Q_{D}(x, d y) Q_{F \cup C}(x, F)>0 .
$$


(Q5) (Intrinsic right continuity) For any $x, F$ and increasing sequence $D_{n}$ with $F \subset D_{n}$, if $Q_{D_{n}}(x, d y) q_{F}(y)$ converges weakly to a finite measure $v(d y)$, then for any $C$ with $F \subset C$ and $f \in b \mathscr{E}_{\Delta}$

$$
Q_{D_{n}}(x, d y) q_{F}(y) 1_{\left[Q_{C}\left(y, f q_{F}\right) \in d a\right]} \rightarrow v(d y) 1_{\left[Q_{C}\left(y, f q_{F}\right) \in d a\right]}
$$

vaguely when restricted as measures on the locally compact $\left(E_{\Delta}-C\right) \times \mathbf{R}$.

(Of course, weak convergence of measures on $E_{\Delta}$ means convergence of integrals of all (real) continuous functions, and vague convergence of measures on a locally compact space means convergence of integrals of all continuous functions vanishing at infinity.)

(Q6) (Quasi-left-continuity) For any $x, D_{n} \downarrow D$ and $F \subset D, Q_{D_{n}}(x, d y) q_{F}(y)$ converges weakly to $Q_{D}(x, d y) q_{F}(y)$.

(Q7) For any $x$ and $D, F$ with $F \subset D^{\circ}, \int Q_{D}(x, d y) q_{F}(y) \log q_{F}(y)<\infty$.

Theorem 1 Let $\left\{Q_{D}(x, \cdot): D \in \mathscr{D}, x \in E_{\Delta}\right\}$ be a family of measures on $E_{\Delta}$ satisfying hypotheses (Q1) through (Q7). Assume $E_{n} \uparrow E$. Then there exists a right process $Y=\left(Y_{t}, P^{x}\right)$ on $E_{\Delta}$ with $\Delta$ as the death point, (which is actually a standard process), and a multiplicative functional $M=\left(M_{t}\right)$ with values in $[0, \infty)$ that is a.s. strictly positive on $\left[0, T_{\Delta}\right), T_{\Delta}=\inf \left\{t \geqq 0: Y_{t}=\Delta\right\}$ the lifetime, such that for all $x \in E, D \in \mathscr{D}$, $f \in b \mathscr{E}_{\Delta}$

$$
Q_{D} f(x)=P^{x}\left[f\left(Y\left(T_{D}\right)\right) M\left(T_{D}\right) ; T_{D}<T_{A}\right]
$$

where $T_{D}=\inf \left\{t \geqq 0: Y_{t} \in D\right\}$.

If $\left\{E_{n}\right\}$ is arbitrary, i.e. does not necessarily contain a subsequence increasing to $E$, then, as in the introduction, we fix for each $n$ an open $U_{n}$ with $\bar{U}_{n} \subset E_{n}$ and denote

$$
\mathscr{D}^{\prime}=\left\{D \in \mathscr{D}: E_{\Delta}-D \subset U_{n} \text { for some } n\right\} .
$$

Theorem 2 Let $\left\{Q_{D}(x, \cdot): D \in \mathscr{D}, x \in E_{\Delta}\right\}$ be a family of measures on $E_{\Delta}$ satisfying hypotheses (Q1) through (Q7). Then there exists a right process $Y=\left(Y_{t}, P^{x}\right)$ on $E_{\Delta}$ with $\Delta$ as the death point, (which is actually a standard process), and a multiplicative functional $M=\left(M_{t}\right)$ with values in $[0, \infty)$ that is a.s. strictly positive on $\left[0, T_{A}\right)$, such that (1.1) is satisfied for all $x \in E, f \in b \mathscr{E}_{A}$ and $D \in \mathscr{D}^{\prime}$.

Of course Theorem 1 is just a special case of Theorem 2. But it is separately stated because it is the important case; also, in Sect. 4 we prove this case first because the proof is easier.

In this article a multiplicative functional $(M F)\left(M_{t}\right)$ in a right process $\left(X_{t}, P^{x}\right)$ on $E_{A}$ is permitted to take values in $[0, \infty)$, not just in $[0,1]$; otherwise it satisfies the usual conditions: $\left(M_{t}\right)$ is adapted to $\left(\mathscr{F}_{t}\right)$, the usual (right continuous and suitable completed) filtration generated by the paths of $\left(X_{t}\right) ; t \rightarrow M_{t}$ is right continuous a.s. (i.e. a.s. $P^{x}$ for all $x \in E$ ); $M_{0}=1$ a.s.; and for any stopping times $T, S$ w.r.t. $\left(\mathscr{F}_{T}\right), M\left(T+S \circ \theta_{T}\right)=M(T) \cdot M(S) \circ \theta_{T}$ a.s. on $\left\{T+S \circ \theta_{T}<\infty\right\}(\theta$ the shift operator). See [2], [6] or [4] for information about standard and right processes.

A MF $\left(\Psi_{t}\right)$ is a transformation (multiplicative) functional (TMF) if $P^{x}\left[\Psi_{t}\right] \leqq 1$ for all $x \in E$ and $t$. It is well-known that if $\left(\Psi_{t}\right)$ is a TMF in a right process 
$\left(X_{t}, P^{x}\right)$ on $E_{\Delta}$, then there exists a right process $\left(\tilde{X}_{t}, \tilde{P}^{x}\right)$ on $E_{\Delta}$ (with $\Delta$ as the death point) such that for all $x, t, f \in b \mathscr{E}_{\Delta}$ (note the convention $f(A)=0$

$$
\tilde{P}^{x}\left[f\left(\tilde{X}_{t}\right)\right]=P^{x}\left[f\left(X_{t}\right) \Psi_{t}\right] .
$$

See e.g. [5]. Such a process $\left(\tilde{X}_{t}\right)$ will be denoted $\left(X_{t} \cdot \Psi_{t}\right)$.

Some remarks need to be made about the hypotheses.

Remarks about (Q2): The universal measurability is a preliminary measurability condition; without it (Q2) and (Q3) cannot be stated. The point $\Delta$ will only serve as the adjoined death point for $\left(Y_{t}\right)$ and the transformed processes $\left(X_{t} \cdot \Psi_{t}\right)$ constructed from the local processes $\left(X_{t}\right)$; thus it carries no mass in the measures $Q_{D}(x, \cdot)$ for $x \in E$. If it is desirable to add mass to the measures $Q_{D}(x, \cdot)$ at an adjoined point, it is preferable to add an isolated point $\partial$ to $E_{\Delta}$ for this purpose, ( $\partial$ is then included in all $D \in \mathscr{D}$ ).

No remarks need to be made about (Q2).

Remarks about (Q3): First, if the conclusion of the theorem (Theorem 2) holds, it is easy to see that $\mathscr{E}_{\Delta}^{n}$ is exactly the $\sigma$-algebra of nearly Borel sets for $\left(Y_{t}\right)$; for $M_{t}>0$ on $\left[0, T_{A}\right)$ a.s. Of course Borel measurability of $\left\{Q_{D}(x, \cdot)\right\}$ implies nearly-Borel measurability, and the latter is a natural measurability assumption. In Sect. 2 we will see that it is equivalent to: $Q_{D}(\cdot, B) \in \mathscr{E}_{\Delta}^{n}$ if $B \in \mathscr{E}_{\Delta}^{n}$.

Remark about (Q4): Under the conclusion of the theorem (Q4) must hold. For if

$$
\int Q_{D}(x, d y) Q_{F \cup C}(y, F)=P^{x}\left[M\left(T_{D}\right) \cdot M\left(T_{F \cup C}\right) \circ \theta\left(T_{D}\right) ; Y\left(T_{F \cup C}\right) \circ \theta\left(T_{D}\right) \in F-\{\Delta\}\right]
$$

(for convenience of writing we use the conventions $Y_{t} \circ \theta_{\infty}=\Delta, Y_{\infty}=\Delta$ and $M_{\infty}=0$ here and below) is 0 for all (sufficiently small) compact neighborhoods $C$ of $x$, then

$$
P^{x}\left[Y\left(T_{F \cup C}\right) \circ \theta\left(T_{D}\right) \in F-\{\Delta\}\right]=0
$$

for all such $C$, and by (Q6) we have

$$
P^{x}\left[Y\left(T_{F \cup\{x\}}\right) \circ \theta\left(T_{D}\right) \in F-\{\Delta\}\right]=0 .
$$

It is then easy to see that $P^{x}\left[Y\left(T_{F}\right) \in F-\{\Delta\}\right]=0$, which implies $Q_{F}\left(x, E_{\Delta}\right)=0$.

Remarks about (Q5): Under the conclusion of the theorem, this condition follows from the intrinsic right continuity of the right process $\left(Y_{t}\right)$, i.e. the right continuity of the filtration $(\mathscr{F})$, relative to which the strong Markov property holds. To see this, first, with $g \in b \mathscr{E}_{A}$ continuous and $T_{n}=T_{D_{n}}$ and $T$ denoting the decreasing limit of $T_{n}$ (of course $T=T_{A}$ where $A=\bigcup_{n} D_{n}$ ), we have

$$
\begin{aligned}
\int Q_{D_{n}}(x, d y) q_{F}(y) g(y) & =P^{x}\left[g\left(Y\left(T_{n}\right)\right) M\left(T_{n}\right) \cdot M\left(T_{F}\right) \circ \theta\left(T_{n}\right)\right] \\
& =P^{x}\left[g\left(Y\left(T_{n}\right)\right) M\left(T_{F}\right)\right] \rightarrow P^{x}\left[g(Y(T)) M\left(T_{F}\right)\right] .
\end{aligned}
$$

Thus $Q_{D_{n}}(x, d y) q_{F}(y)$ converges weakly to $v(d y)=P^{x}\left[M\left(T_{F}\right) ; Y(T) \in d y\right]$ (note these measures all have total mass $\left.q_{F}(x)\right)$. Next, for $f \in b \mathscr{E}_{A}^{+}, y \rightarrow Q_{C}\left(y, f q_{F}\right)$ $=P^{y}\left[f\left(Y\left(T_{C}\right)\right) M\left(T_{F}\right)\right]$ is easily checked to be excessive on $E_{\Delta}-C$ and so a.s. $t \rightarrow Q_{C}\left(Y_{t}, f q_{F}\right)$ is right continuous on $t$-intervals when $Y_{t} \in E_{\Delta}-C$. It follows that $\left.Q_{C}\left(Y\left(T_{n}\right)\right), f q_{F}\right) \rightarrow Q_{C}\left(Y(T), f q_{F}\right)$ a.s. $P^{x}$ on $\left\{Y(T) \in E_{\Delta}-C\right\}$ for $f \in b \mathscr{E}_{\Delta}$. (Actu- 
ally the latter fact is easy to prove directly by martingale convergence.) Thus we have the following vague convergence on $\left(E_{\Delta}-C\right) \times \mathbf{R}$

$$
\begin{aligned}
Q_{D_{n}}(x, d y) q_{F}(y) 1_{\left[Q_{C}\left(y, f q_{F}\right) \in d a\right]} & =P^{x}\left[M\left(T_{F}\right) ; Y\left(T_{n}\right) \in d y, Q_{C}\left(Y\left(T_{n}\right), f q_{F}\right) \in d a\right] \\
& \rightarrow P^{x}\left[M\left(T_{F}\right) ; Y(T) \in d y, Q_{C}\left(Y(T), f q_{F}\right) \in d a\right] \\
& =v(d y) 1_{\left[Q_{C}\left(y, f q_{F}\right) \in d a\right]} .
\end{aligned}
$$

We remark that $Q_{D_{n}}(x, d y) q_{F}(y)$ actually always converges weakly. This follows from the other hypotheses, essentially (Q4) and (Q6); see a remark in Sect. 2 about (H5).

Remarks about (Q6): This condition is satisfied if the underlying right process $\left(Y_{t}\right)$ is a standard process. For if $g \in b \mathscr{E}_{\Delta}$ is continuous, $D_{n} \downarrow D, F \subset D$, then by the quasi-left-continuity of $\left(Y_{t}\right), T_{D_{n}} \uparrow T_{D}$ and $Y\left(T_{D_{n}}\right) \rightarrow Y\left(T_{D}\right)$ on $\left\{T_{F}<T_{\Delta}\right\}$ a.s. $P^{x}$, and so

$$
\begin{aligned}
& \int Q_{D_{n}}(x, d y) q_{F}(y) 9(y) \\
& \quad=P^{x}\left[g\left(Y\left(T_{D_{n}}\right)\right) M\left(T_{F}\right) ; T_{F}<T_{\Delta}\right] \rightarrow P^{x}\left[g\left(Y\left(T_{D}\right)\right) M\left(T_{F}\right) ; T_{F}<T_{A}\right] \\
& \quad=\int Q_{D}(x, d y) q_{F}(y) g(y) .
\end{aligned}
$$

Note that we did not write (Q6) in the following form:

if $D_{n} \downarrow D$ then $Q_{D_{n}}(x, d y)$ converges weakly to $Q_{D}(x, d y)$,

which would reflect the additional requirement that the $\operatorname{MF}\left(M_{t}\right)$ is also "quasileft-continuous".

\section{Local processes}

In this section we fix an (open) set $G \in \mathscr{U}$ and denote $F=G^{c}=E_{\Delta}-G$. Let $\mathscr{D}_{0}$ $=\{D \in \mathscr{D}: F \subset D\}$. We will prove the following theorem.

Theorem 2.1 There exists a right process $\left(X_{t}, P^{x}\right)$ on $E_{\Delta}$ such that for all $D \in \mathscr{D}_{0}$, $x \in E_{\Delta}$ the hitting distribution $P^{x}\left[X\left(T_{D}\right) \in \cdot, T_{D}<\infty\right]$ (where $\left.T_{D}=\inf \left\{t \geqq 0: X_{t} \in D\right\}\right)$ is

$$
H_{D}(x, d y):=Q_{D}(x, d y) q_{F}(x)^{-1} q_{F}(y)
$$

and such that each $x \in F$ is an absorbing point. Furthermore,

$$
J_{t}:=q_{F}\left(X_{0}\right) q_{F}\left(X_{t}\right)^{-1}
$$

defins a MF (with values in $[0, \infty)$ ) such that for all $x, D \in \mathscr{D}_{0}, f \in b \mathscr{E}_{\Delta}$

$$
Q_{D}(x, f)=P^{x}\left[f\left(X\left(T_{D}\right)\right) J\left(T_{D}\right) ; T_{D}<\infty\right] .
$$

The $P^{x}$ in $\left(X_{t}, P^{x}\right)$ is not the same as that in $\left(Y_{t}, P^{x}\right)$ in Theorems $1,2$. Note the measures $H_{D}(x, \cdot)$ are probability measures (since $\int Q_{D}(x, d y) q_{F}(y)$ $=q_{F}(x)$ by $\left.(\mathrm{Q} 2)\right)$ and $H_{D}(x,\{\Delta\})=0$ if $x \neq \Delta$; therefore $T_{F}<\infty$ a.s. and the lifetime $T_{A}=\infty$ a.s. $P^{x}$ for $x \in E$. $\left(T_{F}\right.$ will be called the proper lifetime of $\left(X_{t}\right)$.) For $\left(J_{t}\right)$ to be a MF, one needs only to prove that $J_{t}$ is right continuous a.s. (2.3) is immediate from (2.1) and (2.2). 
For the existence of $\left(X_{t}\right)$ we will need Theorem 1 of [7]. Denote $K=G \cup\left\{\Delta^{*}\right\}$ the one-point compactification of $G . K$ is (obtained from) $E_{\Delta}$ by identifying all points in $F$ as the single point $\Delta^{*}$. $\mathscr{D}_{0}$ is also regarded as the family of compact sets $D$ in $K$ that contain $\Delta^{*}$, i.e. $\left\{(G-U) \cup A^{*}: U \in \mathscr{U}, U \subset G\right\}$ (note that we will often write $\left\{\Delta^{*}\right\}$ as $A^{*}$ ). Thus $H_{D}(x \cdot \cdot)$ in (2.1) is also taken to be a measure on $K$ (when $D \subset K$ ), with of course $H_{D}\left(x, \Delta^{*}\right)$ equal to the original $H_{D}(x, F)$.

With $\left\{H_{D}(x, \cdot): D \in \mathscr{D}_{0}, x \in K\right\}$ regarded as a family of measures on $K$, we will prove that it satisfies hypothesis $(\mathrm{H} 1)$ through $(\mathrm{H} 6)$ in [7], (in doing so $D$ is often regarded as a set in $E_{\Delta}, x$ as in $E_{A}$, and $H_{D}(x, \cdot)$ as a measure on $E_{A}$, and no harm will be done). Denote by $\mathscr{B}$ the $\sigma$-algebra of Borel sets in $K$ and $\mathscr{B}^{*}$ that of universally measurable sets in $K$.

(H1) Each $H_{D}(x, \cdot)$ is a probability measure concentrated on $D ; H_{D}(x, \cdot)=\varepsilon_{x}$ if $x \in D ; H_{D}(\cdot, B) \in \mathscr{B}$ if $B \in \mathscr{B}^{*}$.

Proof. It has been observed that $H_{D}(x, \cdot)$ is a probability measure; the rest is immediate from (Q1).

(H2) (Markov property, or consistency) If $D_{2} \subset D_{1}, H_{D_{2}}(x, \cdot)=\int H_{D_{1}}(x, d y)$ $H_{D_{2}}(y, \cdot)$.

Proof. By (Q2)

$$
\begin{aligned}
H_{D_{2}}(x, d z) & =Q_{D_{2}}(x, d z) q_{F}(x)^{-1} q_{F}(z) \\
& =\int Q_{D_{1}}(x, d y) q_{F}(x)^{-1} q_{F}(y) Q_{D_{2}}(y, d z) q_{F}(y)^{-1} q_{F}(z) \\
& =\int H_{D_{1}}(x, d y) H_{D_{2}}(y, d z) . \quad \square
\end{aligned}
$$

The family $\mathscr{B}^{n}$ of nearly Borel sets of $K$ relative to the family $H_{D}(x, \cdot)$ is defined as follows. A subset $B$ of $K$ is in $\mathscr{B}^{n}$ if for any finite measure $\mu$ on $G$ there exist Borel $B_{1}, B_{2}$, with $B_{1} \subset B \cap G \subset B_{2} \subset G$, such that for all compact $C \subset B_{2}$ $-B_{1}$,

$$
H_{C \cup \Delta^{*}}(\mu, C):=\int \mu(d x) H_{C \cup \Delta^{*}}(x, C)=0 .
$$

Obviously $\mathscr{B} \subset \mathscr{B}^{n} \subset \mathscr{B}^{*}$. From the definitions of $\mathscr{E}_{\Delta}^{n}$ and $\mathscr{B}^{n}$, it is easy to see that $A \in \mathscr{E}_{\Delta}^{n}$ iff $A \cap G \in \mathscr{B}^{n} \cap G$ for all $G \in \mathscr{U}$ (note $\mathscr{B}^{n}$ depends on $G$ ). It is proved in [7, Sect. 2], that $\mathscr{B}^{n}$ is a $\sigma$-algebra; from this the following proposition is immediate.

Proposition $2.2 \mathscr{E}_{\Delta}^{n}$ is a $\sigma$-algebra.

(H3) (Nearly-Borel measurability). $H_{D}(\cdot, B) \in \mathscr{B}^{n}$ if $D \in \mathscr{D}_{0}, B \in \mathscr{B}$.

Proof. Let $f \in b \mathscr{B}^{+}$and $h=H_{D}(\cdot, f)$; we show $h \in \mathscr{B}^{n}$. By a standard argument it suffices to show that, given a finite measure $\mu$ on $G$, there exist $h_{1}, h_{2}$ in $\mathscr{B}^{n}$ with $0 \leqq h_{1} \leqq h \leqq h_{2}$ such that, for all compact $C \subset\left\{h_{2}>h_{1}\right\}$, (2.4) holds. Below we regard $D \subset E_{\Delta}, f \in \mathscr{E}_{\Delta}^{+}$with $f$ constant on $F$, and write $C \cup \Delta^{*}$ as $F \cup C$. Now

$$
h(x)=H_{D}(x, f)=q_{F}(x)^{-1} Q_{D}\left(x, f q_{F}\right) .
$$

Note $q_{F}=Q_{F}\left(\cdot, E_{A}\right) \in \mathscr{E}_{A}^{n}$ by $(\mathrm{Q} 3)$. Define $v(\cdot)=\int \mu(d x) q_{F}(x)^{-1} Q_{D}(x, \cdot)$. Then there exist functions $k_{1}, k_{2}$ in $\mathscr{E}_{4}$ with $0 \leqq k_{1} \leqq q_{F} \leqq k_{2}$ and $k_{1}=q_{F}=k_{2}=1$ on $F$ such 
that for all compact $C_{1} \subset\left\{k_{2}>k_{1}\right\}, Q_{F} \cup C_{1}\left(v, C_{1}\right)=0$. We may change $k_{1}, k_{2}$ on $D^{c}$ to $k_{1}=k_{2}=0$ there. Now let

$$
h_{i}(x)=q_{F}(x)^{-1} Q_{D}\left(x, f k_{i}\right) .
$$

Then $h_{i} \in \mathscr{E}_{A}^{n}$ and $0 \leqq h_{1} \leqq h \leqq h_{2}$. Let us show that for all compact $C \subset\left\{h_{2}>h_{1}\right\}$, $H_{C \cup A^{*}}(\mu, C)=0$, equivalently $Q_{F \cup C}(\mu, C)=0$ (although $Q_{F \cup C}(\mu, \cdot)$ may not be a finite measure). Suppose $Q_{F \cup C}(\mu, C)>0$ for some such $C$; then we claim

$$
Q_{F \cup C_{1}}\left(\mu, C_{1}\right)>0 \text { for some compact } C_{1} \subset\left\{k_{2}>k_{1}\right\} \text {. }
$$

But this leads to a contradiction since $C_{1} \subset D$ and so

$$
Q_{F \cup C_{1}}\left(\mu, C_{1}\right)=\int Q_{D}(\mu, d y) Q_{F \cup C_{1}}\left(y, C_{1}\right)=0,
$$

which follows from $Q_{F \cup c_{1}}\left(v, C_{1}\right)=0$, a consequence of the choice of $k_{i}$. To show (2.5), let $C^{\prime}=C \cap D$; then since obviously $\left\{h_{2}>h_{1}\right\} \cap D \subset\left\{k_{2}>k_{1}\right\}, C^{\prime} \subset\left\{k_{2}>k_{1}\right\}$. By $(\mathrm{Q} 2)$ we have

$$
Q_{F \cup C^{\prime}}\left(\mu, C^{\prime}\right)=\int Q_{F \cup C}(\mu, d y) Q_{F \cup C^{\prime}}\left(y, C^{\prime}\right) .
$$

If $Q_{F \cup C}\left(\mu, C^{\prime}\right)>0$ then $Q_{F \cup C^{\prime}}\left(\mu, C^{\prime}\right)>0$. Otherwise $Q_{F \cup C}\left(\mu, C-C^{\prime}\right)>0$. But if $y \in C-C^{\prime}$, then since $h_{2}(y)>h_{1}(y), Q_{D}\left(y,\left\{k_{2}>k_{1}\right\}\right)>0$, and so

$$
Q_{F \cup C^{\prime}}\left(y, C^{\prime}\right)=\int Q_{D}(y, d z) Q_{F \cup C^{\prime}}\left(z, C^{\prime}\right)>0
$$

if $C^{\prime}$ is sufficiently large (i.e. $C$ is sufficiently large). Thus (2.5) follows, where $C_{1}$ is $C^{\prime}$ when $C$ is sufficiently large.

It is proved in [7, Sect. 2], that $H_{D}(\cdot, B) \in \mathscr{B}^{n}$ for $D \in \mathscr{D}_{0}, B \in \mathscr{B}^{n}$, using an argument resembling the above. In the present situation, we can prove the fact (not needed in the sequel) that $Q_{D}(\cdot, B) \in \mathscr{E}_{\Delta}^{n}$ for $D \in \mathscr{D}, B \in \mathscr{E}_{\Delta}^{n}$, using a similar but slightly more careful argument. Of course, once we obtain a process $\left(Y_{t}\right)$, this also follows from a standard fact in a right process.

(H4) (Quasi-left-continuity) If $D_{n} \downarrow D, H_{D_{n}}(x, \cdot)$ converges weakly to $H_{D}(x, \cdot)$.

Proof. This is immediate from (Q6) and the definition of $H_{D}(x, \cdot)$.

(H5) (Intrinsic right continuity) For any $x$ in $K$, increasing sequence $D_{n}$ in $\mathscr{D}_{0}$, and letting $\left(W_{n}\right)$ be the nonhomogeneous reversed Markov chain on $K$ (under a single probability measure $P$ ) with $P\left(W_{n} \in \cdot\right)=H_{D_{n}}(x, \cdot)$ and $P\left(W_{n+1} \in \cdot \mid W_{m}, m \leqq n\right)=H_{D_{n+1}}\left(W_{n}, \cdot\right)$, we have: if $W_{\infty}=\lim W_{n}$ exists a.s., then, for any $C \in \mathscr{D}_{0}$ and $f \in b \mathscr{B}, H_{C} f\left(W_{n}\right) \rightarrow H_{C} f\left(W_{\infty}\right)$ a.s. on $\left\{W_{\infty} \in K-C\right\}$.

Proof. We first show that (H5) follows from condition (H5) below and then prove $\left(\mathrm{H} 5^{\prime}\right)$ from $(\mathrm{Q} 5)$.

$\left(\mathrm{H} 5^{\prime}\right)$. For $x, D_{n}$ as in (H5), if $H_{D_{n}}(x, \cdot)$ converges weakly (as measures on $K$ ) to a measure $\mu$, then for any $C, f$ as in (H5) the measures

$$
H_{D_{n}}(x, d y) 1_{\left[H_{C}(y, f) \in d a\right]} \rightarrow \mu(d y) 1_{\left[H_{C}(y, f) \in d a\right]}
$$

vaguely when restricted to $(K-C) \times \mathbf{R}$. 
If $W_{n} \rightarrow W_{\infty}$ a.s. in (H5), then $H_{D_{n}}(x, \cdot)$ converges weakly to $\mu(\cdot)=P\left(W_{\infty} \in \cdot\right)$. Now by an easy application of martingale convergence $H_{C} f\left(W_{n}\right)$ converges a.s. on $\left\{W_{\infty} \in K-C\right\}$. The conclusion in $\left(\mathrm{H} 5^{\prime}\right)$ says the measures

$$
P\left(W_{n} \in d y, H_{C}\left(W_{n}, f\right) \in d a\right) \rightarrow P\left(W_{\infty} \in d y, H_{C}\left(W_{\infty}, f\right) \in d a\right)
$$

vaguely when restricted to $\left\{W_{\infty} \in K-C\right\}$. From this it is clear that the conclusion of (H5) follows. To prove $\left(\mathrm{H} 5^{\prime}\right)$, assume $H_{D_{n}}(x, \cdot)$ converges weakly to $\mu$ as measures on $K$; then we claim that $Q_{D_{n}}(x, d y) q_{F}(y)=q_{F}(x) H_{D_{n}}(x, \cdot)$ converges weakly as measures on $E_{\Delta}$ (which has a coarser topology at the boundary $\partial G$ ). For if not, it is not difficult to construct a sequence $D_{n}^{\prime} \downarrow D^{\prime}$ where $F \subset D^{\prime}$ such that $Q_{D_{n}^{\prime}}(x, d y) q_{F}(y)$ does not converge weakly, and so (Q6) is contradicted. (We do not give the detail of this part of the proof since we feel that it would be quite reasonable to assume in (Q5) that $Q_{D_{n}}(x, d y) q_{F}(y)$ converges weakly. Incidentally, it was proved in [7] that the $\left(W_{n}\right)$ in $(\mathrm{H} 5)$ always converges weakly, essentially by $(\mathrm{H} 6)$ below; therefore also does $H_{D_{n}}(x, \cdot)$ in $\left(\mathrm{H} 5^{\prime}\right)$. In this article, it can also be proved that $Q_{D_{n}}(x, d y) q_{F}(y)$ in (Q 5) always converges weakly, using (Q4) and (Q6).) Let $v$ be the weak limit of $Q_{D_{n}}(x, d y) q_{F}(y)$; then $\mu(d y)$ $=q_{F}(x)^{-1} v(d y)$ with the understanding $\mu\left\{\Lambda^{*}\right\}=q_{F}(x)^{-1} v(F)$. Applying (Q5) with $C=F$ and $f=1$ we have

$$
Q_{D_{n}}(x, d y) q_{F}(y) 1_{\left[q_{F}(y) \in d a\right]} \rightarrow v(d y) 1_{\left[q_{F}(y) \in d a\right]}
$$

vaguely on $\left(E_{A}-F\right) \times \mathbf{R}$. Combining this with the vague convergence of

$$
Q_{D_{n}}(x, d y) q_{F}(y) 1_{\left[Q_{C}\left(y, f q_{F}\right) \in d a\right]} \rightarrow v(d y) 1_{\left[Q_{\mathrm{C}}\left(y, f q_{F}\right) \in d a\right]}
$$

on $\left(E_{\Delta}-C\right) \times \mathbf{R}$ for the given $f$, and noting that $q_{F}(y)>0$, we obtain

$$
Q_{D_{n}}(x, d y) q_{F}(y) 1_{\left[q_{F}(y)^{-1} Q_{C}\left(y, f q_{F}\right) \in d a\right]} \rightarrow v(d y) 1_{\left[q_{F}(y)^{-1} Q_{C}\left(y, f q_{F}\right) \in d a\right]}
$$

vaguely on $\left(E_{A}-C\right) \times \mathbf{R}$. Dividing the above by $q_{F}(x)$ we have the conclusion of $\left(\mathrm{H}^{\prime}\right)$.

(H6) (Transience) For any $D \in \mathscr{D}_{0}$ and $x \notin D$, there exists a compact neighborhood $C$ of $x$ such that $\int H_{D}(x, d y) H_{C \cup A^{*}}(y, C)<1$.

Proof. This is immediate from (Q4) and the fact that the $H_{D}(y, \cdot)$ are probability measures.

Now that (H1) though (H6) are established, it follows from Theorem 1 of [7] that there exists a right process (actually a Hunt process) $\left(\bar{X}_{t}, P^{x}\right)$ on $K$ with $A^{*}$ as the death point and with finite lifetime $T_{\Delta^{*}}$ such that the hitting distributions are the given $H_{D}(x, \cdot)$. The process $\left(X_{t}, P^{x}\right)$ in Theorem 2.1 can be obtained as follows. One shows that for $f \in b_{\mathscr{E}_{A}^{+}}^{+}, \lim _{t \uparrow T_{\Delta^{*}}} H_{F} f\left(\bar{X}_{t}\right)$ exists a.s. $P^{x}$ for each $x \in G$ and has $P^{x}$-expectation $H_{F} f(x)$ (because $H_{F} f$ is excessive and in fact harmonic on $G$ ). Thus we can define $\left(X_{t}, t<T_{F}\right)$ as $\left(\bar{X}_{t}, t<T_{\Delta^{*}}\right)$, and then define the distributions $P^{x}\left[X\left(T_{F}\right) \in d y \mid X_{t}, t<T_{F}\right]$ by requiring $P^{x}\left[f\left(X\left(T_{F}\right)\right) \mid X_{t}, t\right.$ $\left.<T_{F}\right]=\lim _{t \uparrow T_{F}} H_{F} f\left(X_{t}\right)$ for all continuous $f$. Then since each $y \in F$ is to be an absorbing point, the existence of $\left(X_{t}\right)$ as desired follows. 
However, there is an easier way to define $\left(X_{t}\right)$. Let $X_{t}^{1}$ be the process obtained from $\left(X_{t}\right)$ by changing each $x \in F-\Delta$ to a holding point, from which a jump is made to $\Delta$ with probability 1 . We know what the hitting distributions $H_{D}^{1}(x, \cdot)$ should be. Let $\mathscr{D}_{\Delta}=\left\{D: D\right.$ is closed in $\left.E_{\Delta}, \Delta \in D\right\}$. Define $\left\{H_{D}^{1}(x, \cdot): D \in \mathscr{D}_{\Delta}, x \in E_{\Delta}\right\}$ as follows:

$$
H_{D}^{1}(x, \cdot)=\varepsilon_{x}(\cdot) \quad \text { if } x \in D ; \quad H_{D}^{1}(x, \cdot)=\varepsilon_{A}(\cdot) \quad \text { if } x \in F-D ;
$$

and otherwise

$$
\begin{aligned}
H_{D}^{1}(x, B) & =\int H_{F \cup D}(x, d y) H_{D}^{1}(y, B) \\
& =H_{F \cup D}(x, D \cap B)+1_{B}(\Delta) H_{F \cup D}(x, F-D) .
\end{aligned}
$$

(In particular, if $F \subset D, H_{D}^{1}(x, \cdot)=H_{D}(x, \cdot)$.) It is easy to see that $\left\{H_{D}^{1}(x, \cdot)\right\}$ satisfies (H1) through (H6). Thus there exists a right process $\left(X_{t}^{1}\right)$ on $E_{\Delta}$ with the $H_{D}^{1}(x, \cdot)$ as its hitting distributions. Now change each holding, point $x \in F-\Delta$ to an absorbing point to obtain $\left(X_{t}\right)$ from $\left(X_{t}^{1}\right)$.

To complete the proof of Theorem 2.1, it remains to show $\left(J_{t}\right)$ defined is (2.2) is a MF. Note $J_{0}=1$ and $J_{t+s}=J_{t} \cdot J_{s}\left(\theta_{t}\right)$ for all $t, s$. So one needs only to show $J_{t}$ is right continuous a.s. This of course will follow from

Proposition $2.3 q_{F}\left(X_{t}\right)$ is right continuous a.s.

Proof. By a standard theorem it suffices to show $q_{F}$ is nearly Borel and finely continuous w.r.t. $\left(X_{t}\right)$. We have already that $q_{F}$ is nearly Borel. Since each $x \in F$ is absorbing, to prove that $q_{F}$ is finely continuous we need only to show the fine continuity at each $x \in G$. Define $A=\left\{y \in G:\left|q_{F}(y)-q_{F}(x)\right| \geqq \delta\right\}$ where $\delta>0$. We show that $P^{x}\left(T_{A}>0\right)=1$. Suppose not; then $P^{x}\left(T_{A}=0\right)=1$. Let $C_{n}$ be increasing compact subsets of $A$ such that $T_{C_{n}} \downarrow T_{A}$ a.s. $P^{x}$. Let $D_{n}=C_{n} \cup F$. Then $Q_{D_{n}}(x, d y) q_{F}(y)=q_{F}(x) P^{x}\left[X\left(T_{D_{n}}\right) \in d y\right] \quad$ converges weakly to $v(d y)$ $=q_{F}(x) P^{x}\left[X\left(T_{A}\right) \in d y\right]=q_{F}(x) \varepsilon_{x}(d y)$. By (Q5) with $C=F$ and $f=1$ we thus have that $q_{F}\left(X\left(T_{D_{n}}\right)\right)$ under $P^{x}$ converges in distribution to $Q_{F}\left(x, q_{F}\right)=Q_{F}(x, 1)=q_{F}(x)$, which is a contradiction.

\section{The multiplicative functional connecting two local processes}

From now on a right process $\left(X_{t}, P^{x}\right)$ is simply written as $X_{t}$ and a MF $\left(M_{t}\right)$ as $M_{t}$. We will occasionally write the probability measure $P_{x}^{i}$ in $\left(X_{t}^{i}, P_{x}^{i}\right)$ as $P^{x}$. All (right) processes $X_{t}$ will have as sample space the space $\Omega$ of right continuous paths $\omega:[0, \infty) \rightarrow E_{\Delta}$ such that $\omega_{t}=\Delta$ implies $\omega_{s}=\Delta$ for $s>t$; furthermore we can require all $\omega$ to have left limits on $\left(0, T_{\Delta}\right)$ where $T_{\Delta}=\inf \left\{t: \omega_{t}=A\right\}$. Of course $X_{t}(\omega)=\omega_{t}$, and the same notation $T_{D}=\inf \left\{t \geqq 0: X_{t} \in D\right\}$ is therefore used for all $X_{t}$. A MF $M_{t}$ (in a process $X_{t}$ ) is said to be "trajectory-dependent" (more appropriately trajectory-dependent-only) if for any finite measure $\mu$ on $E_{\Delta}$ there exists $A$ such that $P^{\mu}\left(\Lambda^{c}\right)=0$ and for all $\omega_{1}, \omega_{2}$ in $A$ and $t_{1}, t_{2}>0$, and increasing homeomorphism $\sigma:\left[0, t_{1}\right] \rightarrow\left[0, t_{2}\right]$ with $X_{t}\left(\omega_{1}\right)=X_{\sigma(t)}\left(\omega_{2}\right)$ on $\left[0, t_{1}\right]$, we have $M_{t}\left(\omega_{1}\right)=M_{\sigma(t)}\left(\omega_{2}\right)$ on $\left[0, t_{1}\right]$. All MFs will be trajectory-dependent, but we will still usually mention the fact explicitly for emphasis. If $U$ is an open set in $E$, a MF $M_{t}$ is said to be "constant off $U$ " if a.s. the following holds: for any $t_{1}<t_{2}$, if $X_{t}(\omega) \in U^{c}$ for all $t \in\left[t_{1}, t_{2}\right]$ then $M_{t}(\omega)$ is constant on $\left[t_{1}, t_{2}\right]$. 
If two processes $X_{t}^{1}$ and $X_{t}^{2}$ are time changes of each other (which is the case iff they have the same hitting distributions), we will write $X_{t}^{1} \sim X_{t}^{2}$. If $U$ is an open set in $E$, the process $Z_{t}$ obtained by stopping $X_{t}$ at the exit from $U$, (i.e. $Z_{t}=X_{t \wedge T}$ where $T=T_{U c}$ ), will be written as $\left.X_{t}\right|_{U}$. In this section we prove the following theorem that is of central importance.

Theorem 3.1 Let $G_{1}, G_{2}$ and $U$ be in $\mathscr{U}$ with $\bar{U} \subset G_{1} \subset G_{2}$. Let $X_{t}^{i}$ be the right process $X_{t}$ defined in Sect. 2 when $G=G_{i}, i=1,2$. Then there exists a TMF $\Psi_{t}$ in $X_{t}^{2}$ such that $\left.\left.X_{t}^{2} \cdot \Psi_{t}\right|_{U} \sim X_{t}^{1}\right|_{U}$. Furthermore, $\Psi_{t}$ satisfies the following properties:

(i) it is trajectory-dependent;

(ii) it is constant off $U$;

(iii) a.s. $\Psi_{t}$ is strictly positive on $\left[0, T_{A}\right)$.

Let $F_{i}=G_{i}^{\mathrm{c}}, q_{i}=q_{F_{i}}$, and denote $\zeta_{i}=T_{F_{i}}$ (again for both $X_{t}^{1}$ and $X_{t}^{2}$ ), the proper lifetime of $X_{t}^{i}$. The hitting distributions of $X_{t}^{i}$ for $D \in \mathscr{D}$ containing $F_{i}$ are

$$
H_{D}^{i}(x, d y)=Q_{D}(x, d y) q_{i}(x)^{-1} q_{i}(y)
$$

and with the MF $J_{t}^{i}$ in $X_{t}^{i}$ defined by

$$
J_{t}^{i}=q_{i}\left(X_{0}^{i}\right) q_{i}\left(X_{t}^{i}\right)^{-1}
$$

we have, for $x \in E, D \in \mathscr{D}$ containing $F_{i}, f \in b \mathscr{E}_{\Delta}$

$$
Q_{D} f(x)=P^{x}\left[f\left(X^{i}\left(T_{D}\right)\right) J^{i}\left(T_{D}\right)\right] .
$$

We will regard $J_{t}^{1}$ as also defined in $X_{t}^{2}$ by $J_{t}^{1}=q_{1}\left(X_{0}^{2}\right) q_{1}\left(X_{t}^{2}\right)^{-1}$. Denote $Z_{t}$ $=\left.X_{t}^{2}\right|_{G_{1}}$. The following defines a MF in $Z_{t}$ :

$$
\phi_{t}=J_{t \wedge \zeta_{1}}^{2} / J_{t_{\wedge} \zeta_{1}}^{1} .
$$

Denote by $\mathscr{F}_{t}^{2}$ the filtration generated by the paths of $X_{t}^{2}$ that is, as always, right continuous and completed in the usual way. Of course $\phi_{t}$ is adapted to $\mathscr{F}_{t}^{2}$.

Lemma 3.2 (i) Let $T$, $S$ be $\mathscr{F}_{T}^{2}$-stopping times with $T \leqq S \leqq \zeta_{1}$ and $\rho \in\left(\mathscr{F}_{T}^{2}\right)^{+}$; then

$$
P_{x}^{2}[\rho \phi(S) / \phi(T)]=P_{x}^{2}[\rho]
$$

for all $x$. $Z_{t}$.

(ii) If $S$ is as above, $P_{x}^{2}[\phi(S)]=1$ for all $x$; consequently $\phi_{t}$ is a TMF in (iii) $Z_{t} \cdot \phi_{t} \sim X_{t}^{1}$.

Proof. (i) First, by (3.1), (3.2) and (3.3)

$$
P_{y}^{2}\left[\phi\left(\zeta_{1}\right)\right]=\int Q_{F_{1}}(y, d z) q_{2}(y)^{-1} q_{2}(z)\left[q_{2}(y) q_{2}(z)^{-1}\right] /\left[q_{1}(y) q_{1}(z)^{-1}\right]=1,
$$

since $q_{F_{1}}(y)=Q_{F_{k}}\left(y, q_{F_{1}}\right)$. Thus

$$
\begin{aligned}
P_{x}^{2}[\rho \phi(S) / \phi(T)] & =P_{x}^{2}\left[\rho \cdot \frac{\phi(S)}{\phi(T)} \cdot P_{X(S)}^{Z}\left(\phi\left(\zeta_{1}\right)\right)\right]=P_{x}^{2}\left[\rho \cdot \frac{\phi\left(\zeta_{1}\right)}{\phi(T)}\right] \\
& =P_{x}^{2}\left[\rho \phi\left(\zeta_{1}\right) \circ \theta_{T}\right]=P_{x}^{2}\left[\rho P_{X(T)}^{2}\left(\phi\left(\zeta_{1}\right)\right)\right]=P_{x}^{2}[\rho] .
\end{aligned}
$$


(ii) follows from (i) with $\rho=1, T=0$.

(iii) will follow if $Z_{t} \cdot \phi_{t}$ and $X_{t}^{1}$ have the same hitting distributions on sets $D \in \mathscr{D}$ containing $F_{1}$, noting that each $x \in F_{1}$ is an absorbing point for both processes. But by a computation similar to the above, the hitting distributions of $Z_{t} \cdot \phi_{t}$ for such $D$ are

$$
P_{x}^{2}\left[f\left(X^{2}\left(T_{D}\right)\right) \phi\left(T_{D}\right)\right]=\int Q_{D}(x, d y) f(y) q_{1}(x)^{-1} q_{1}(y)=H_{D}^{1}(x, f) .
$$

We will construct the part of the TMF $\Psi_{t}$ in Theorem 3.1 up to time $\zeta_{1}$ (i.e. its part in $Z_{t}$ ); its part after time $\zeta_{1}$ will be determined by the requirement that $\Psi_{t}$ be a $\mathrm{MF}$ and be constant off $U$, noting $\bar{U} \subset G_{1}$ and $\zeta_{1}=T_{F_{1}}$, (the part of $\Psi_{t}$ up to $\zeta_{1}$ has this latter property). To this end choose compact sets $C_{n}$, $n \geqq 0$, with $C_{n} \uparrow U$ and $C_{0}=\emptyset$ (the empty set), denote $V=U^{c}$, and define, for $n \geqq 1, \mathscr{F}_{t}^{2}$-stopping times $T_{n j}, R_{n j}, j \geqq 0$, and $S_{n j}, j \geqq 1$, as follows:

$$
\begin{aligned}
& T_{n 0}=0, \quad R_{n 0}=T_{V}\left(=\inf \left\{t \geqq 0: X_{t}^{2} \in V\right\}\right), \quad \text { and for } j \geqq 1 \\
& T_{n j}=R_{n, j-1}+T_{C_{n} \cup F_{1}} \circ \theta\left(R_{n, j-1}\right), \\
& S_{n j}=T_{n j}+T_{C_{n-1} \cup V^{\circ}} \theta\left(T_{n j}\right), \\
& R_{n j}=S_{n j}+T_{V^{\circ}} \theta\left(S_{n j}\right)=T_{n j}+T_{V^{\circ}} \theta\left(T_{n j}\right) .
\end{aligned}
$$

Since paths (of $X_{t}^{2}$ ) have left limits on $\left(0, T_{A}\right.$ ), we have for a fixed $n, T_{n j}=S_{n j}$ $=R_{n j}=\zeta_{1}$ for all sufficiently large $j$. Note the intervals $\left[T_{n j}, S_{n j}\right), n \geqq 1, j \geqq 1$, (most of them being $\emptyset$ ), are disjoint, and their union is $\left(T_{V}, \zeta_{1}\right) \cap\left\{t: X_{t}^{2} \in U\right\}$. Note also $\left[T_{n j}, R_{n j}\right), j \geqq 1$, is $\left[T_{n j}, S_{n j}\right)$ or its union with some $\left[S_{n-1, i}, R_{n-1, i}\right)$. Define

$$
\Phi_{n}(t)=\prod_{j=0}^{\infty} \frac{\phi\left(R_{n j} \wedge t\right)}{\phi\left(T_{n j} \wedge t\right)}=\prod_{j=0}^{\infty} \phi\left(T_{V} \wedge\left(t-T_{n j}\right)^{+}\right) \circ \theta\left(T_{n j}\right)
$$

(where in the last expression both $T_{n j}$ are evaluated at $\omega$ ). Note the products are finite, and $\Phi_{n}(t)=\Phi_{n}\left(t \wedge \zeta_{1}\right) . \Phi_{n}(t)$ is not a MF (in $\left.Z_{t}\right)$, but satisfies the following properties: $\Phi_{n}(0)=1 ; \Phi_{n}(t)$ is right continuous; $\Phi_{n}(t+s)=\Phi_{n}(t) \cdot \Phi_{n}(s) \circ \theta_{t}$ for all $t, s$ with $t<\zeta_{1}$ and $X_{t}^{2} \notin U-C_{n}$; it is trajectory-dependent; it is constant off $U$ and $\Phi_{n}(t)>0$ for all $t$. Observe that

$$
\frac{\Phi_{n}(t)}{\Phi_{n-1}(t)}=\prod_{j=1}^{\infty} \frac{\phi\left(S_{n j} \wedge t\right)}{\phi\left(T_{n j} \wedge t\right)}=\prod_{j=1}^{\infty} \phi\left(T_{C_{n-1} \cup V} \wedge\left(t-T_{n j}\right)^{+}\right) \circ \theta\left(T_{n j}\right) .
$$

Lemma $3.3 P_{x}^{2}\left[\Phi_{n}(S)\right] \leqq 1$ for all $x$ and $\mathscr{F}_{t}^{2}$-stopping time $S \leqq \zeta_{1}$.

Proof. $\Phi_{n}(S)$ is the limit, as $j^{\prime} \rightarrow \infty$, of $\prod_{j=0}^{j^{\prime}} \phi\left(R_{n j} \wedge S\right) / \phi\left(T_{n j} \wedge S\right)$, whose $P_{x}^{2}$-expectation is $P_{x}^{2}[1]=1$ by repeatedly using (i) of Lemma 3.2.

Lemma $3.4 P_{x}^{2}\left[\log \phi\left(\zeta_{1}\right)\right]>-\infty$ for all $x$.

Proof.

$$
\begin{aligned}
P_{x}^{2}\left[\log \phi\left(\zeta_{1}\right)\right] & =\int Q_{F_{1}}(x, d y) q_{2}(x)^{-1} q_{2}(y) \log \left[q_{2}(x) q_{2}(y)^{-1} q_{1}(x)^{-1} q_{1}(y)\right] \\
& =\int Q_{F_{1}}(x, d y) q_{2}(x)^{-1} q_{2}(y)\left[\log \left(q_{2}(x) q_{1}(x)^{-1}\right)-\log q_{2}(y)\right] \\
& =\log \left(q_{2}(x) q_{1}(x)^{-1}\right)-q_{2}(x)^{-1} \int Q_{F_{1}}(x, d y) q_{2}(y) \log q_{2}(y)>-\infty
\end{aligned}
$$


by applying (Q7) with $D=F_{1}, F=F_{2}$; in the second equality we used $q_{1}=1$ on $F_{1}$.

Theorem 3.5 For all $x$ and $\delta>0, \sup _{n_{2}>n_{1}} P_{x}^{2}\left[\sup _{t}\left|\frac{\Phi_{n_{2}}(t)}{\Phi_{n_{1}}(t)}-1\right|>\delta\right] \rightarrow 0$ as $n_{1} \rightarrow \infty$.

Proof. Suppose not. Then for some $x$ and $\delta>0$ there exist $1 \leqq m_{1}<m_{1}^{\prime} \leqq \ldots \leqq m_{I}$ $<m_{l}^{\prime} \leqq \ldots$ such that for all $l$

$$
P_{x}^{2}\left[\sup _{t}\left|\frac{\Phi_{m_{i}}(t)}{\Phi_{m_{l}}(t)}-1\right|>\delta\right]>\delta .
$$

Fix $l$ and denote $n_{1}=m_{l}, n_{2}=m_{l}^{\prime}$. In the above $t$ can be restricted to belonging to

$$
\bigcup_{n_{1}<n \leqq n_{2}} \bigcup_{1 \leqq j \leqq j_{0}}\left[T_{n j}, S_{n j}\right)
$$

for some $j_{0}$. Let $\gamma=\inf \left\{t:\left|\frac{\Phi_{n_{2}}(t)}{\Phi_{n_{1}}(t)}-1\right| \geqq \delta\right\}$; note that if $\gamma<\infty$, the inf is a.s. a minimum by the a.s. right continuity of $\phi(t)$ and therefore of $\Phi_{n}(t)$. Arrange the disjoint intervals $\left[T_{n j} \wedge \gamma, S_{n j} \wedge \gamma\right.$ ), $n_{1}<\leqq n_{2}, 1 \leqq j \leqq j_{0}$, (some of which may be empty), as $\left[\tau_{1}, \sigma_{1}\right), \ldots,\left[\tau_{k^{\prime}}, \sigma_{k^{\prime}}\right)$, where $k^{\prime}=j_{0}\left(n_{2}-n_{1}\right)$, with $\tau_{1} \leqq \sigma_{1} \leqq \ldots \leqq \tau_{k^{\prime}}$ $\leqq \sigma_{k^{\prime}} \leqq \zeta_{1}$ being $\mathscr{\mathscr { F }}_{t}^{2}$-stopping times. By (3.4)

$$
P_{x}^{2}\left[\left|\frac{\Phi_{n_{2}}\left(\sigma_{k^{\prime}}\right)}{\Phi_{n_{1}}\left(\sigma_{k^{\prime}}\right)}-1\right| \geqq \delta\right]>\delta .
$$

Now $\Phi_{n_{2}}\left(\sigma_{k^{\prime}}\right) / \Phi_{n_{1}}\left(\sigma_{k^{\prime}}\right)=\prod_{k \leqq k^{\prime}} \phi\left(\sigma_{k}\right) / \phi\left(\tau_{k}\right)$. From Lemma 3.2(i) (see Lemma 3.3)

$$
P_{x}^{2}\left[\prod_{k \leqq k^{\prime}} \phi\left(\sigma_{k}\right) / \phi\left(\tau_{k}\right)\right]=1 .
$$

Combining the above we have

$$
P_{x}^{2}\left[\log \prod_{k \leqq k^{\prime}} \phi\left(\sigma_{k}\right) / \phi\left(\tau_{k}\right)\right]<\varepsilon
$$

where $\varepsilon>0$ depends only on $\delta$. Denote the above $k^{\prime}$ by $k_{l}$, and $\tau_{k}, \sigma_{k}$ by $\tau_{l k}, \sigma_{l k}$, (recall $\left.n_{1}=m_{l}, n_{2}=m_{l}^{\prime}\right)$. The intervals $\left[\tau_{l k}, \sigma_{l k}\right), 1 \leqq k \leqq k_{l}, 1 \leqq l \leqq l_{1},\left(l_{1}\right.$ arbitrary), are disjoint. Let the complement in $\left[0, \zeta_{1}\right)$ of their union be the union of disjoint intervals $\left[\tau_{0 k}, \sigma_{0 k}\right), 1 \leqq k \leqq k_{0}$, (again some may be empty), where $\tau_{01} \leqq \sigma_{01}$ $\underset{\text { Now }}{\leqq} \leqq \tau_{0 k_{0}} \leqq \sigma_{0 k_{0}}$ are $\mathscr{\mathscr { F }}_{t}^{2}$-stopping times. Of course $P_{x}^{2}\left[\prod_{k \leqq k_{0}} \phi\left(\sigma_{0 k}\right) / \phi\left(\tau_{0 k}\right)\right]=1$.

$$
\phi\left(\zeta_{1}\right)=\phi\left(\zeta_{1}\right) / \phi(0)=\prod_{l=0}^{l_{1}} \prod_{k=1}^{k_{l}} \phi\left(\sigma_{l k}\right) / \phi\left(\tau_{l k}\right)
$$

So by (3.5) and Lemma 3.4

$$
-\infty<P_{x}^{2}\left[\log \phi\left(\zeta_{1}\right)\right]=\sum_{l=0}^{l_{1}} P_{x}^{2}\left[\log \prod_{k=0}^{k_{2}} \phi\left(\sigma_{k l} / \sigma\left(\tau_{k l}\right)\right]<-l_{1} \varepsilon .\right.
$$

This is a contradiction since $l_{1}$ is arbitrary. 


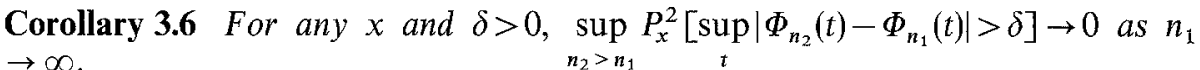

Proof. Fix $n$ and $b>0$ and let $S=\zeta_{1} \wedge \inf \left\{t: \Phi_{n}(t)>b\right\}$. Then $\Phi_{n}(S) \geqq b$ if $\sup \Phi_{n}(t)>b$. Since $P_{x}^{2}\left[\Phi_{n}(S)\right] \leqq 1$ by Lemma $3.3, P_{x}^{2}\left[\sup \Phi_{n}(t)>b\right] \leqq 1 / b$ for all $n$. Thus

$$
P_{x}^{2}\left[\sup _{t}\left|\Phi_{n_{2}}(t)-\Phi_{n_{1}}(t)\right|>\delta\right] \leqq P_{x}^{2}\left[\sup _{t}\left|\frac{\Phi_{n_{2}}(t)}{\Phi_{n_{1}}(t)}-1\right|>\delta / b\right]+1 / b
$$

and the corollary follows from Theorem 3.5.

Definition. For each $x$ let integers $1=n_{1}(x)<\ldots<n_{k}(x)<\ldots$ be defined inductively by

$$
n_{k+1}(x)=\inf \left\{n>n_{k}(x): \sup _{n^{\prime}>n} P_{x}^{2}\left[\sup _{t}\left|\Phi_{n^{\prime}}(t)-\Phi_{n}(t)\right|>2^{-k}\right]<2^{-k}\right\}
$$

(the $n_{k}(x)$ exist by Corollary 3.6); then set

$$
\Phi_{\infty}(t)=\frac{\lim }{k} \Phi_{n_{k}\left(X_{0}^{2}\right)}(t)
$$

Theorem 3.7 (i) A.s. ( $P_{\mu}^{2}$ for any finite measure $\mu$ ) $\Phi_{n_{k}(X z)}(t) \rightarrow \Phi_{\infty}(t)$ uniformly in $t$.

(ii) A.s. $\Phi_{\infty}(t)$ is right continuous; $\Phi_{\infty}(0)=1 ; \Phi_{\infty}(t)=\Phi_{\infty}\left(t \wedge \zeta_{1}\right) ; \Phi_{\infty}(t)$ is adapted to $\mathscr{F}_{t}^{2} ; \Phi_{\infty}(t)$ is trajectory-dependent, and is constant off $U$.

(iii) $P_{x}^{2}\left[\Phi_{\infty}(S)\right] \leqq 1$ for any $\mathscr{F}_{t}^{2}$-stopping time $S \leqq \zeta_{1}$.

(iv) For $\mathscr{F}_{t}^{2}$-stopping time $T, S$ with $T+S \circ \theta_{T} \leqq \zeta_{1}, \Phi_{\infty}\left(T+S \circ \theta_{T}\right)=\Phi_{\infty}(T)$ . $\Phi_{\infty}(S) \circ \theta_{T}$ a.s.

(v) $\left.\left.Z_{t} \cdot \Phi_{\infty}(t)\right|_{U} \sim X_{t}^{1}\right|_{U},\left(\right.$ from the above $\Phi_{\infty}(t)$ is a TMF in $\left.Z_{t}\right)$.

(vi) A.s. $\Phi_{\infty}(t)$ is strictly positive on $\left[0, \zeta_{1}\right]$ ( or on $[0, \infty)$ ).

Proof. (i) follows form the definition of $n_{k}$ (x). (ii) follows from (i), the corresponding properties of $\Phi_{n}(t)$ and the fact that $\left\{x: n_{k}(x)=n\right\} \in \mathscr{E}_{A}^{*}$. (iii) follows from (i) and Lemma 3.3. To show (iv) holding a.s. $P_{x}^{2}$, choose a subsequence $n_{k(i)}(x)$ of $n_{k}(x)$ such that a.s. $P_{x}^{2}, n_{k(i)}(x)>n_{i}\left(X_{T}^{2}\right)$ for all sufficiently large $i$; then using the fact that, if $n(i)>n_{i}(y)$ for all large $i, \Phi_{n(i)}(t)$ and $\Phi_{n_{i}(y)}(t)$ converge uniformly in $t$ to the same limit a.s. $P_{y}^{2}$, and using the fact $\left.\Phi_{n}(T+S) \circ \theta_{T}\right)=\Phi_{n}(T) \cdot \Phi_{n}(S) \circ \theta_{T}$ except possibly on $\left\{X_{T}^{2} \in U-C_{n}\right\}$, which set $\downarrow \emptyset$ since $C_{n} \uparrow U$, one obtains the desired equality. (v) follows from Lemma 3.2(iii) since $\Phi_{\infty}(t)=\Phi_{n}(t)=\phi(t)$ for $t \leqq T_{V}=T_{E_{\Delta}-U}$. It remains to show (vi). Let

$$
\hat{\Phi}_{n}(t)=\phi(t) / \Phi_{n}(t) .
$$

Then we can write

$$
\hat{\Phi}_{n}(t)=\prod_{j=1}^{\infty} \frac{\phi\left(\tau_{n j} \wedge t\right)}{\phi\left(\gamma_{n j} \wedge t\right)}
$$


where $\gamma_{n 1} \leqq \tau_{n 1} \leqq \ldots \leqq \gamma_{n j} \leqq \tau_{n j} \leqq \ldots$ are $\mathscr{F}_{t}^{2}$-stopping times with $\gamma_{n j}=\tau_{n j}=\zeta_{1}$ for all sufficiently large $j$; in fact the union of $\left[\gamma_{n j}, \tau_{n j}\right)$ is $\left[0, \zeta_{1}\right)-\bigcup_{j=0}^{\infty}\left[T_{n j}, R_{n j}\right)$. As is with $\Phi_{n}(t)$ we have $P_{x}^{2}\left[\hat{\Phi}_{n}(S)\right] \leqq 1$ for $\mathscr{F}_{t}^{2}$-stopping times $S \leqq \zeta_{1}$. Since $\hat{\Phi}_{n_{2}}(t) / \hat{\Phi}_{n_{1}}(t)=\Phi_{n_{1}}(t) / \Phi_{n_{2}}(t)$, Theorem 3.5 implies that for all $x$ and $\delta>0$

$$
\sup _{n_{2}>n_{1}} P_{x}^{2}\left[\sup _{t}\left|\hat{\Phi}_{n_{2}}(t) / \hat{\Phi}_{n_{1}}(t)-1\right|>\delta\right] \rightarrow 0 \quad \text { as } n_{1} \rightarrow \infty .
$$

It follows that Corollary 3.6 also holds for $\hat{\Phi}_{n}(t)$. Let $\hat{n}_{k}(x)$ be defined in a similar way to $n_{k}(x)$ but with the additional requirement of being a subsequence of $n_{k}(x): \hat{n}_{1}(x)=1$ and

$$
\begin{aligned}
\hat{n}_{k+1}(x)= & \inf \left\{n>\hat{n}_{k}(x): n=n_{i}(x) \text { for some } i,\right. \\
& \left.\sup _{n^{\prime}>n} P_{x}^{2}\left[\sup _{t} \widehat{\Phi}_{n^{\prime}}(t)-\widehat{\Phi}_{n}(t) \mid>2^{-k}\right]<2^{-k}\right\} .
\end{aligned}
$$

Define $\widehat{\Phi}_{\infty}(t)=\lim _{k} \widehat{\Phi}_{\hat{n}_{k}\left(X_{0}^{2}\right)}(t)$. Then a.s. $\widehat{\Phi}_{\hat{n}_{k}\left(X_{0}^{2}\right)}(t) \rightarrow \widehat{\Phi}_{\infty}(t)$ uniformly in $t$; so a.s.

$$
\phi(t)=\lim _{k} \Phi_{\hat{n}_{k}\left(X_{0}^{2}\right)}(t) \hat{\Phi}_{\hat{n}_{k}\left(X_{0}^{2}\right)}(t)=\Phi_{\infty}(t) \hat{\Phi}_{\infty}(t) \quad \text { on }\left[0, \zeta_{1}\right] .
$$

Since $\phi(t)$ is strictly positive on $\left[0, \zeta_{1}\right]$, (vi) follows.

We now define $\Psi_{t}$ on $\left[0, \zeta_{1}\right]$ by

$$
\Psi_{t}=\Phi_{\infty}(t), \quad t \leqq \zeta_{1}
$$

and extend to $t \in[0, \infty)$ by the requirement that $\Psi_{t}$ be a MF (in $X_{t}^{2}$ ) and be constant off $U$ (using the fact that $\Psi_{t}, t \leqq \zeta_{1}$, is constant off $U$ ). To make this definition precise, let $T_{n}$ be defined by $T_{0}=0$ and

$$
\begin{aligned}
T_{2 k+1} & =T_{2 k}+T_{\bar{U}^{\circ}} \theta\left(T_{2 k}\right), & & k \geqq 0 ; \\
T_{2 k} & =T_{2 k-1}+T_{F_{1}} \circ \theta\left(T_{2 k-1}\right), & & k \geqq 1 .
\end{aligned}
$$

Of course $T_{n}=\infty$ for sufficiently large $n$. Define

$$
\begin{aligned}
\Psi_{t} & =\prod_{k=0}^{\infty} \Phi_{\infty}\left(\zeta_{1} \wedge\left(t-T_{2 k+1}\right)^{+}\right) \circ \theta\left(T_{2 k+1}\right) \\
& = \begin{cases}\prod_{k=0}^{m-1} \Phi_{\infty}\left(\zeta_{1}\right) \circ \theta\left(T_{2 k-1}\right) & \text { if } T_{2 m} \leqq t \leqq T_{2 m+1} \\
\Psi\left(T_{2 m-1}\right) \cdot \Phi_{\infty}\left(t-T_{2 m+1}\right) \circ \theta\left(T_{2 m+1}\right) & \text { if } T_{2 m+1}<t<T_{2 m+2}\end{cases}
\end{aligned}
$$

(an empty product stands for 1 ).

Proof of Theorem 3.1 From Theorem 3.7 and the definition of $\Psi_{t}$ we have: a.s. $\Psi_{t}$ is right continuous, $\Psi_{0}=1, \Psi_{t}>0$ on $[0, \infty)$, and $\Psi_{t}$ is constant off $U$ (note $\Psi_{t}$ is constant on $\left[T_{2 m}, T_{2 m+1}\right]$ ). It is routine to show that $\Psi_{t}$ is adapted to $\mathscr{F}_{t}^{2}$, and that for two $\mathscr{F}_{t}^{2}$-stopping times $T, S$ we have $\Psi\left(T+S \circ \theta_{T}\right)$ 
$=\Psi(T) \cdot \Psi(S) \circ \theta_{T}$ a.s. We now show $P_{x}^{2}\left[\Psi_{t}\right] \leqq 1$ for all $x, t$. For this it suffices to show $P_{x}^{2}\left[\Psi\left(t \wedge T_{n}\right)\right] \leqq 1$. Now

$$
P_{x}^{2}\left[\Psi\left(t \wedge T_{n}\right)\right]=P_{x}^{2}\left[\Psi(t) ; t<T_{n-1}\right]+P_{x}^{2}\left[\Psi\left(t \wedge T_{n}\right) ; t \geqq T_{n-1}\right] .
$$

The second term on the right hand side is $P_{x}^{2}\left[\Psi\left(T_{n-1}\right) ; t \geqq T_{n-1}\right]$ if $n-1$ is even, (since $\Psi_{t}$ is constant on $\left[T_{2 m}, T_{2 m+1}\right]$ ), and if $n-1$ is odd it is

$$
\begin{aligned}
P_{x}^{2} & {\left[\Psi\left(T_{n-1}\right) \cdot \Phi_{\infty}\left(\zeta_{1} \wedge\left(t-T_{n-1}\right)\right) \circ \theta\left(T_{n-1}\right) ; t \geqq T_{n-1}\right] } \\
& =P_{x}^{2}\left[\Psi\left(T_{n-1}\right) P_{X^{2}\left(T_{n-1}\right)}^{2}\left\{\Phi_{\infty}\left(\zeta_{1} \wedge\left(t-T_{n-1}\right)\right)\right\} ; t \geqq T_{n-1}\right] \\
& \leqq P_{x}^{2}\left[\Psi\left(T_{n-1}\right) ; t \geqq T_{n-1}\right]
\end{aligned}
$$

by Theorem 3.7 (iii). Therefore,

$$
P_{x}^{2}\left[\Psi\left(t \wedge T_{n}\right)\right] \leqq P_{x}^{2}\left[\Psi\left(t \wedge T_{n-1}\right)\right] \leqq \ldots \leqq P_{x}^{2}\left[\Psi\left(t \wedge T_{1}\right)\right] \leqq 1
$$

Thus $\Psi_{t}$ is a TMF in $X_{t}^{2}$. Finally, the assertion $\left.\left.X_{t}^{2} \cdot \Psi_{t}\right|_{U} \sim X_{t}^{1}\right|_{U}$ is just Theorem $3.7(\mathrm{v})$.

\section{The global process and associated multiplicative functional}

We prove Theorem 1 using two methods. The first method is simpler but not quite rigorous (but perhaps could satisfy some readers); the second method is involved but rigorous. The two methods are not essentially different in the case of Theorem 1. The proof of Theorem 2 uses an extension of the second method.

\subsection{First proof of Theorem 1}

Here $E_{m} \uparrow E$. We may assume $E_{m} \subset \bar{E}_{m} \subset E_{m+1}$. Choose $U_{m} \in \mathscr{U}$ such that $E_{m-1} \subset \bar{U}_{m} \subset E_{m}$. Denote $F_{m}=E_{m}^{c}, q_{m}=q_{F_{m}}$ and $\mathscr{D}_{m}=\left\{D \in \mathscr{D}: F_{m} \subset D\right\}$. Let $X_{t}^{m}$ be the right process $X_{t}$ constructed in Sect. 2 with $G=E_{m}$, and $J_{t}^{m}$ be the MF in all $X_{t}^{N}, N \geqq m$, defined by

Then

$$
J_{t}^{m}=q_{m}\left(X_{0}^{N}\right) q_{m}\left(X_{t}^{N}\right)^{-1}
$$

$$
Q_{D}(x, f)=P_{x}^{m}\left[f\left(X^{m}\left(T_{D}\right)\right) J^{m}\left(T_{D}\right)\right], \quad x \in E_{A}, \quad D \in \mathscr{D}_{m} .
$$

Let $\zeta_{m}=T_{F_{m}}$. Let $\Psi_{t}^{m}$ be the TMF (in $X_{t}^{m+1}$ ) constructed in Sect. 3 with $G_{1}=E_{m}$, $G_{2}=E_{m+1}, U=U_{m}$ and $X_{t}^{1}, X_{t}^{2}$ there being $X_{t}^{m}, X_{t}^{m+1}$. Thus

$$
\Psi_{t}^{m}=J_{t}^{m+1} / J_{t}^{m}=\frac{q_{m+1}}{q_{m}}\left(X_{0}^{m+1}\right) \frac{q_{m}}{q_{m+1}}\left(X_{t}^{m+1}\right), \quad \text { for } t \leqq T_{U_{m}^{c}}
$$

and

$$
\left.\left.X_{t}^{m+1} \cdot \Psi_{t}^{m}\right|_{U_{m}} \sim X_{t}^{m}\right|_{U_{m}}
$$


Now the TMF $\Psi_{t}^{m-1}$ in $X_{t}^{m}$ depends only on the trajectories of $\left.X_{t}^{m}\right|_{E_{m-1}}$ and is constant off $U_{m-1}$. Since $E_{m-1} \subset U_{m},(4.3)$ implies that the trajectory-dependent $\Psi_{t}^{m-1}$ can be regarded as a TMF in $X_{t}^{m+1} \cdot \Psi_{t}^{m}$, first defined up to time $\zeta_{m-1}$, then to all $t$ by the requirement that it be a MF and be constant off $U_{m-1}$, by the procedure at the end of Sect. 3, (note a slight abuse of notation is involved because this $\Psi_{i}^{m-1}$ should be written as $\Psi_{i}^{m+1, m-1}$ ). Thus we can form the transformed process $\left(X_{t}^{m+1} \cdot \Psi_{t}^{m}\right) \cdot \Psi_{t}^{m-1}$, which will be written as $X_{t}^{m+1} \cdot \Psi_{t}^{m} \Psi_{t}^{m-1}$. (It is not difficult to argue that $\Psi_{t}^{m-1}$ exists as a MF in $X_{t}^{m+1}$ and $\Psi_{t}^{m} \Psi_{t}^{m-1}$ is a TMF. However we will use a different method in Subsect. 4.2 to construct directly (in $X_{t}^{m+1}$ ) TMF's $\Psi_{t}^{m} \Psi_{t}^{m-1}$ and $\Psi_{t}^{m} \Psi_{t}^{m-1} \ldots \Psi_{t}^{k}$ below.) It satisfies

$$
\left.\left.X_{t}^{m+1} \cdot \Psi_{t}^{m} \Psi_{t}^{m-1}\right|_{U_{m-1}} \sim X_{t}^{m-1}\right|_{U_{m-1}},\left.\left.\quad X_{t}^{m+1} \cdot \Psi_{t}^{m} \Psi_{t}^{m-1}\right|_{U_{m}} \sim X_{t}^{m} \cdot \Psi_{t}^{m-1}\right|_{U_{m}} .
$$

Proceeding in this manner we construct processes

$$
\left(\ldots\left(X_{t}^{m+1} \cdot \Psi_{t}^{m}\right) \ldots\right) \cdot \Psi_{t}^{k}=X_{t}^{m+1} \cdot \Psi_{t}^{m} \ldots \Psi_{t}^{k}
$$

(again $\Psi_{t}^{j}$ in the above should be written as $\Psi_{t}^{m+1, j}$ ), which satisfy

$$
\left.\left.X_{t}^{m+1} \cdot \Psi_{t}^{m} \ldots \Psi_{t}^{k}\right|_{U_{k}} \sim X_{t}^{k}\right|_{U_{k}}
$$

and more importantly

$$
\left.\left.X_{t}^{m+1} \cdot \Psi_{t}^{m} \ldots \Psi_{t}^{k}\right|_{U_{m}} \sim X_{t}^{m} \cdot \Psi_{t}^{m-1} \ldots \Psi_{t}^{k}\right|_{U_{m}} .
$$

Define right processes $Y_{t}^{m}$ on $E_{\Delta}$ by

$$
Y_{t}^{1}=\left.X_{t}^{1}\right|_{U_{1}} ; \quad Y_{t}^{m}=\left.X_{t}^{m} \cdot \Psi_{t}^{m-1} \ldots \Psi_{t}^{1}\right|_{U_{m}}, \quad m \geqq 2 .
$$

Since each $X_{t}^{m}$ is a Hunt process, each $Y_{t}^{m}$ is a standard process; we again call $U_{m}$ the proper state space of $Y_{t}^{m}$ (each $x \in V_{m}=U_{m}^{c}$ is absorbing), and its proper lifetime $\zeta_{m}^{\prime}=T_{U_{m}^{c}}$ is finite. By (4.4)

$$
\left.Y_{t}^{m+1}\right|_{U_{m}} \sim Y_{t}^{m}, \quad m \geqq 1 .
$$

From the theorem in [8], (4.5) implies that there exists a right process $Y_{t}$ on $E_{\Delta}$ (which is a standard process with lifetime $T_{\Delta}=\lim _{m} \zeta_{m}^{\prime}$ ) such that

$$
\left.Y_{t}\right|_{U_{m}} \sim Y_{t}^{m}, \quad m \geqq 1 .
$$

Let us define the desired MF $M_{t}$ in $Y_{t}$. First, if $X_{t}$ is a right process on $E_{A}$ with death point $\Delta$ and if $\Psi_{t}$ is a TMF in $X_{t}$ that is strictly positive on $\left[0, T_{A}\right.$ ), then $\Psi_{t}^{-1} \cdot 1_{\left[t<T_{\Delta}\right]}$, which we simply write as $\Psi_{t}^{-1}$, is a MF of the transformed process $\widetilde{X}_{t}=X_{t} \cdot \Psi_{t}$, (noting that both $X_{t}$ and $\tilde{X}_{t}$ have sample space $\Omega-$ see the beginning paragraph of Sect. 3, and we regard $\Psi_{t}$ as defined in $\widetilde{X}_{t}$ as well with the understanding (or requirement) that if $\omega_{1}(s)=\omega_{2}(s) \in E$ for all $s \in[0, t]$ then $\left.\Psi_{t}\left(\omega_{1}\right)=\Psi_{t}\left(\omega_{2}\right)\right)$, and

$$
\tilde{P}^{x}\left[f\left(X_{t} \cdot \Psi_{t}\right) \Psi_{t}^{-1}\right]=P^{x}\left[f\left(X_{t}\right) \Psi_{t} \Psi_{t}^{-1}\right]=P^{x}\left[f\left(X_{t}\right)\right], \quad f \in b \mathscr{E}_{\Delta} \text { (with } f(\Delta)=0 \text { ). }
$$


Now in $X_{t}^{m} \cdot \Psi_{t}^{m-1} \ldots \Psi_{t}^{1}$ we have the $\operatorname{MF}\left(\Psi_{t}^{m-1} \ldots \Psi_{t}^{1}\right)^{-1}$, (again the TMF $\Psi_{t}^{m-1} \ldots \Psi_{t}^{1}$ is to be rigorously defined in Subsect. 4.2), which satisfies

$$
\begin{aligned}
\tilde{P}_{x}^{m} & {\left[f\left(X_{t}^{n} \cdot \Psi_{t}^{m-1} \ldots \Psi_{t}^{1}\right)\left(\Psi_{t}^{m-1} \ldots \Psi_{t}^{1}\right)^{-1}\right] } \\
& =P_{x}^{m}\left[f\left(X_{t}^{m}\right)\left(\Psi_{t}^{m-1} \ldots \Psi_{t}^{1}\right)\left(\Psi_{t}^{m-1} \ldots \Psi_{t}^{1}\right)^{-1}\right]=P_{x}^{m}\left[f\left(X_{t}\right)\right] .
\end{aligned}
$$

Define $M_{t}^{m}$ by

$$
M_{t}^{1}=J_{t \wedge \zeta_{1}^{\prime}}^{1} ; \quad M_{t}^{m}=J_{t \wedge \zeta_{m}^{\prime}}^{m}\left(\Psi_{t \wedge \zeta_{m}^{m}}^{m-1} \ldots \Psi_{t \wedge \zeta_{m}^{\prime}}^{1}\right)^{-1}, \quad m \geqq 2 .
$$

$M_{t}^{m}$ is a $\mathrm{MF}$ in $Y_{t}^{m}$. We have the consistency

$$
M_{t}^{m+1}=M_{t}^{m}, \quad t \leqq \zeta_{m}^{\prime}
$$

since by (4.2)

$$
J_{t}^{m+1}\left(\Psi_{t}^{m}\right)^{-1}=J_{t}^{m+1}\left(J_{t}^{m+1} / J_{t}^{m}\right)^{-1}=J_{t}^{m}, \quad t \leqq \zeta_{m}^{\prime} .
$$

Since all MFs are trajectory-dependent, (4.6) implies that $M_{t}^{m}$ can be regarded as defined in $Y_{t}$. The consistency (4.9) permits the definition

$$
\begin{aligned}
M_{t} & =M_{t}^{m} & & \text { on }\left[0, \zeta_{m}^{\prime}\right], \quad m \geqq 1 \\
& =0 & & \text { on }\left[T_{\Delta}=\underset{m}{=} \lim _{m}, \infty\right] .
\end{aligned}
$$

$M_{t}$ is obviously a MF in $Y_{t}$. It remains to show equality (1.1). For $x \in E, D \in \mathscr{D}$ (so $U_{m}^{c} \subset D$ for some $m$ ), $f \in b \mathscr{E}_{A}$, and with $P^{x}, P_{x}^{m}, \widehat{P}_{x}^{m}$ denoting the probability measures for $Y_{t}, X_{t}^{m}, Y_{t}^{m}$ and writing $T=T_{D}$, (also noting $M_{t}=0$ on $\left[T_{\Delta}, \infty\right]$ )

$$
\begin{aligned}
P^{x}\left[f\left(Y_{T}\right) M_{T}\right] & =\hat{P}_{x}^{m}\left[f\left(Y_{T}^{m}\right) M_{T}^{m}\right]=\hat{P}_{x}^{m}\left[f\left(Y_{T}^{m}\right) J_{T}^{m}\left(\Psi_{T}^{m-1} \ldots \Psi_{T}^{1}\right)^{-1}\right] \\
& =P_{x}^{m}\left[f\left(X_{T}^{m}\right)\left(\Psi_{T}^{m-1} \ldots \Psi_{T}^{1}\right) J_{T}^{m}\left(\Psi_{T}^{m-1} \ldots \Psi_{T}^{1}\right)^{-1}\right] \\
& =P_{x}^{m}\left[f\left(X_{T}^{m}\right) J_{T}^{m}\right]=Q_{D} f(x)
\end{aligned}
$$

by (4.10), (4.5), the definition of $Y_{t}^{m}$, the computation (4.7), and (4.1).

\subsection{Second proof of Theorem 1}

Fix $N \geqq 2$. We will construct directly in $X_{t}^{N}$ MFs $\Psi_{t}^{m}, 1 \leqq m<N$, that appeared in 4.1 , and show that $\Psi_{t}^{N-1} \ldots \Psi_{t}^{m}$ are TMFs. Let $C_{m n}$ be compact sets with $C_{m 0}=\emptyset, C_{m n} \uparrow U_{m}$ as $n \rightarrow \infty$ and $C_{m n}$ increasing in $m$ for each $n$. With $X_{t}^{N}, X_{t}^{m}$ (respectively $X_{t}^{m+1}$ ), $E_{N}, E_{m}$ (respectively $E_{m+1}$ ), and $U_{m}$ playing the roles of $X_{t}^{2}, X_{t}^{1}, G_{2}, G_{1}$ and $U$ in Sect. 3, and with $C_{m n}=C_{n}$, we obtain a TMF $\bar{\Psi}_{t}^{N m}$ (respectively $\widetilde{\Psi}_{t}^{N m}$ ) that is the TMF $\Psi_{t}$ in Sect. 3 . (Note $\widetilde{\Psi}_{t}^{N, N-1} \equiv 1$.) Thus

$$
\left.\left.X_{t}^{N} \cdot \bar{\Psi}_{t}^{N m}\right|_{U_{m}} \sim X_{t}^{m}\right|_{U_{m}},\left.\left.\quad X_{t}^{N} \cdot \tilde{\Psi}_{t}^{N m}\right|_{U_{m}} \sim X_{t}^{m+1}\right|_{U_{m}} .
$$

Recall the approximating functionals $\Phi_{n}(t)$ for $\Phi_{\infty}(t)=\Psi_{t \wedge \zeta_{1}}$ in Sect. 3; if we extend the definition of $\Phi_{n}(t)$ to $t \leqq \zeta_{2}$, by replacing $\zeta_{1}=T_{F_{1}}$ by $\zeta_{2}=T_{F_{2}}$ in the definition of $\Phi_{n}(t)$, then obviously we have a.s. $\Phi_{n_{k}\left(X_{0}^{2}\right)}(t) \rightarrow \Psi(t)$ uniformly on $\left[0, \zeta_{2}\right]$ (or on $[0, \infty)$ ), where $n_{k}(x)$ is similarly defined (see below). Let $\bar{\Phi}_{n}^{N m}(t)$ (respectively $\widetilde{\Phi}_{n}^{N m}(t)$ ) denote the approximating functional $\Phi_{n}(t)$ when $\Psi(t)$ is the 
above $\bar{\Psi}^{N m}(t)$ (respectively $\widetilde{\Psi}^{N m}(t)$ ), (they will be explicitly defined in the proof of Theorem 4.1 below). Thus a.s. in $X_{t}^{N}$

$$
\bar{\Phi}_{n_{k}\left(X_{0}^{N}\right)}^{N m}(t) \rightarrow \bar{\Psi}^{N m}(t), \quad \tilde{\Phi}_{n_{k}\left(X_{0}^{N}\right)}^{N m} \rightarrow \widetilde{\Psi}^{N m}(t)
$$

uniformly in $t$ on $\left[0, \zeta_{N}\right]$, where $n_{1}(x)=1$ and

$$
\begin{aligned}
n_{k+1}(x)= & \inf \left\{n>n_{k}(x): \sup _{n^{\prime}>n} P_{x}^{N}\left[\sup _{t}\left|\bar{\Phi}_{n^{\prime}}^{N m}(t)-\bar{\Phi}_{n}^{N m}(t)\right|>2^{-k}\right]<2^{-k}\right. \text {, and } \\
& \left.\sup _{n^{\prime}>n} P_{x}^{N}\left[\sup _{t}\left|\widetilde{\Phi}_{n^{\prime}}^{N m}(t)-\widetilde{\Phi}_{n}^{N m}(t)\right|>2^{-k}\right]<2^{-k} \text {, for } 1 \leqq m<N\right\} .
\end{aligned}
$$

Since a.s. $\widetilde{\Psi}^{N m}(t)>0$ for all $t$, we can define

$$
\Psi^{m}(t)=\Psi^{N m}(t)=\bar{\Psi}^{N m}(t) / \tilde{\Psi}^{N m}(t) .
$$

$\Psi^{m}(t)$ is a MF (but not a TMF) in $X_{t}^{N}$. We have a.s. $\bar{\Phi}_{n}^{N m}(t) / \tilde{\Phi}_{n}^{N m}(t) \rightarrow \Psi^{m}(t)$ uniformly on $\left[0, \zeta_{N}\right]$, as $n=n_{k}\left(X_{0}^{N}\right) \rightarrow \infty$.

Theorem 4.1 $\Psi_{t}^{N-1} \Psi_{t}^{N-2} \ldots \Psi_{t}^{m}$ is a TMF in $X_{t}^{N}$ for $1 \leqq m<N$.

Proof. We need only to prove the case $m=1$. Let

$$
L_{n}(t)=\prod_{m=1}^{N-1} \bar{\Psi}_{n}^{N m}(t) / \tilde{\Phi}_{n}^{N m}(t)
$$

It suffices to prove that for any $x$ and $\mathscr{F}_{t}^{N}$-stopping time $S \leqq \zeta_{N}$, we have $P_{x}^{N}\left[L_{n}(S)\right] \leqq 1$. Define for $1 \leqq m_{1}<m_{2} \leqq N$

$$
\phi_{t}^{m_{2} m_{1}}=J_{t}^{m_{2}} / J_{t}^{m_{1}}=\left(q_{m_{2}} q_{m_{1}}^{-1}\right)\left(X_{0}^{N}\right)\left(q_{m_{2}}^{-1} q_{m_{1}}\right)\left(X_{t}^{N}\right)
$$

and to simplify expressions write for $r \leqq s$ (and only when $X_{t}^{N} \in U_{m_{1}}$ for $r \leqq t<s$ )

$$
\phi_{t}^{m_{2} m_{1}}[r, s)=\phi^{m_{2} m_{1}}(s) / \phi^{m_{2} m_{2}}(r) .
$$

Define $\mathscr{F}_{t}^{N}$-stopping times $T_{n j}^{m}, R_{n j}^{m}, 1 \leqq m<N, n \geqq 1, j \geqq 0$ as follows (for a fixed $m$, these are the stopping times $T_{n j}, R_{n j}$ in Sect. 3 when $C_{m n}=C_{n}$ and $U_{m}=U$, but with $\zeta_{1}=T_{F_{1}}$ there replaced by $\zeta_{2}=T_{F_{2}}$; here $\zeta_{2}$ is $\zeta_{N}=T_{F_{N}}$ ), where $V_{m}=U_{m}^{c}$ :

$$
\begin{aligned}
T_{n 0}^{m} & =0 ; \quad R_{n j}^{m}=T_{n j}^{m}+T_{V_{m}} \circ \theta\left(T_{n j}^{m}\right) ; \\
T_{n, j+1}^{m} & =R_{n j}^{m}+T_{F_{N} \cup C_{m n}} \circ \theta\left(R_{n, j}^{m}\right) .
\end{aligned}
$$

Again, for sufficiently large $j, T_{n j}^{m}=R_{n j}^{m}=\zeta_{N}$. Note $\left[T_{n j}^{m}, R_{n j}^{m}\right) \subset\left\{t: X_{t}^{N} \in U_{m}\right\}$. Now for $m \leqq m_{1} \leqq m_{2} \leqq N$ define

$$
\begin{aligned}
\Phi_{n}^{m_{2} m_{1} m}(x) & =\prod_{j=0}^{\infty} \phi^{m_{2} m_{1}}\left[T_{n j}^{m} \wedge t, R_{n j}^{m} \wedge t\right), \\
\Phi_{n}^{m_{2} m}(t) & =\Phi^{m_{2} m m}(t) .
\end{aligned}
$$

(Note $\bar{\Phi}_{n}^{N m}(t)=\Phi_{n}^{N m m}(t), \widetilde{\Phi}_{n}^{N m}(t)=\Phi_{n}^{N, m+1, m}(t)$, and so since $\phi_{t}^{m+1, m}=\phi_{t}^{N m} / \phi_{t}^{N, m+1}$ we have a.s.

$$
\left.\Phi_{n}^{m+1, m}(t)=\Phi_{n}^{N m m}(t) / \Phi_{n}^{N, m+1, m}(t) \rightarrow \Psi^{m}(t), \text { as } n=n_{k}\left(X_{0}^{N}\right) \rightarrow \infty .\right)
$$


Now define $\mathscr{F}_{t}^{N}$-stopping times $\tau_{n j}^{m}, \gamma_{n j}^{m}$ with $\tau_{n 0}^{m} \leqq \gamma_{n 0}^{m} \leqq \ldots \leqq \tau_{n j}^{m} \leqq \gamma_{n j}^{m} \leqq \ldots$ such that $\left[\tau_{n j}^{1}, \gamma_{n j}^{1}\right)=\left[T_{n j}^{1}, R_{n j}^{1}\right)$, and for $m \geqq 2, \bigcup_{j}\left[\tau_{n j}^{m}, \gamma_{n j}^{m}\right)=\bigcup_{j}\left[T_{n j}^{m}, R_{n j}^{m}\right)-\bigcup_{j}\left[T_{n j}^{m-1}\right.$, $R_{n j}^{m-1}$ ), (note $\bigcup_{j}\left[T_{n j}^{m}, R_{n j}^{m}\right)$ is increasing in $m$ ). We have

$$
\begin{aligned}
L_{n}(t) & =\prod_{m=1}^{N-1} \Phi_{n}^{m+1, m}(t)=\prod_{m=1}^{N-1} \prod_{j=1}^{\infty} \phi^{m+1, m}\left[T_{n j}^{m} \wedge t, R_{n j}^{m} \wedge t\right) \\
& =\prod_{m=1}^{N-1} \prod_{j=0}^{\infty} \prod_{k=m}^{N-1} \phi^{k+1, k}\left[\tau_{n j}^{m} \wedge t, \gamma_{n j}^{m} \wedge t\right) \\
& =\prod_{m=1}^{N-1} \prod_{j=1}^{\infty} \phi^{N m}\left[\tau_{n j}^{m} \wedge t, \gamma_{n j}^{m} \wedge t\right) .
\end{aligned}
$$

As alway this is a finite product. As in Lemma 3.3, to show $P_{x}^{N}\left[L_{n}(S) \leqq 1\right]$ is suffices to prove

$$
P_{x}^{N}\left[\prod_{m=1}^{N-1} \prod_{j=1}^{j_{1}} \phi^{N m}\left[\tau_{n j}^{m} \wedge S, \gamma_{n j}^{m} \wedge S\right)\right] \leqq 1
$$

As in Lemma 3.2, for $\mathscr{F}_{i}^{N}$-stopping times $T_{1} \leqq T_{2} \leqq \zeta_{N}$ with $\left[T_{1}, T_{2}\right) \subset\left\{t: X_{t}^{N} \in U_{m}\right\}$, and $\rho \in \mathscr{F}^{N}\left(T_{1}\right)^{+}$

$$
P_{x}^{N}\left[\rho \phi^{N m}\left(T_{2}\right) / \phi^{N m}\left(T_{1}\right)\right]=P_{x}^{N}[\rho]
$$

whose proof relies only on the fact that $P_{y}^{N}\left[\phi^{N m}\left(\zeta_{m}\right)\right]=1$ for $y \in E_{m}$. By rearranging the stochastic intervals $\left[\tau_{n j}^{m} \wedge S, \gamma_{n j}^{m} \wedge S\right)$ in all possible increasing orders and applying (4.15) repeatedly to the probability in (4.14), we obtain (4.14).

We now have the transformed processes $X_{t}^{N} \cdot \Psi_{t}^{N-1} \ldots \Psi_{t}^{1}$. They satisfy

$$
\left.\left.X_{t}^{N+1} \cdot \Psi_{t}^{N} \Psi_{t}^{N-1} \ldots \Psi_{t}^{1}\right|_{U_{m}} \sim X_{t}^{N} \cdot \Psi_{t}^{N-1} \ldots \Psi_{t}^{1}\right|_{U_{m}} .
$$

For $\left.\left.X_{t}^{N+1} \cdot \Psi_{t}^{N}\right|_{U_{m}} \sim X_{t}^{N}\right|_{U_{m}}$ and in these processes, $\Psi_{t}^{N-1} \ldots \Psi_{t}^{1}$ are the "same" trajectory-dependent functional; alternatively, one can argue directly that for $U_{m}^{c} \subset D, f \in b \mathscr{E}_{\Delta}$

$$
P_{x}^{N+1}\left[f\left(X_{T}^{N+1}\right) \Psi_{T}^{N} \Psi_{T}^{N-1} \ldots \Psi_{T}^{1}\right]=P_{x}^{N}\left[f\left(X_{T}^{N}\right) \Psi_{T}^{N-1} \ldots \Psi_{T}^{1}\right]
$$

where $T=T_{D}$. The rest of the proof of Theorem 1 (involving the definition of $Y_{t}^{N}, Y_{t}, M_{t}^{N}$ and $M_{t}$ ) is identical to that in Subsect. 4.1 (after (4.4)).

\subsection{Proof of Theorem 2}

Assume as we may that the $E_{m}$ are all distinct, $\left\{E_{m}\right\}$ is closed w.r.t. finite nonempty intersection, and $E_{n} \notin E_{m}$ if $m<n$. Choose open $U_{m}$ with $\bar{U}_{m} \subset E_{m}$ such that $\left\{U_{m}\right\}$ is a covering of $E, U_{m} \subset U_{n}$ if $E_{m} \subset E_{n}$, and $U_{m}=U_{m_{1}} \cap \ldots \cap U_{m_{l s}}$ if $E_{m}$ $=E_{m_{1}} \cap \ldots \cap E_{m_{k}}$. Let compact sets $C_{m n} \uparrow U_{m}$ with $C_{m 0}=\emptyset$ and satisfy $C_{m n}$ $=C_{m_{1} n} \cap \ldots \cap C_{m_{k} n}$ if $E_{m}=E_{m_{1}} \cap \ldots \cap E_{m_{k}}$. 
We write $m_{1} \dot{<} m_{2}$ if $E_{m_{1}} \subset E_{m_{2}}$ and $m_{1}<m_{2}$ (so that $E_{m_{1}} \neq E_{m_{2}}$ ). If $m_{1}<m_{2}$, we write $m_{2} \backslash m_{1}=1$ if there exists no $m$ with $m_{1} \dot{<} m \dot{<} m_{2}$ and $m_{2} \backslash m_{1}>1$ otherwise. If $m_{1} \dot{<} m_{2}$, a "route" connecting $m_{1}$ and $m_{2}$ is a sequence $\left(n_{1}, \ldots, n_{k}\right)$ with $m_{1}=n_{1} \dot{<} n_{2} \dot{<} \ldots \dot{<} n_{k}=m_{2}$ such that $n_{i+1} \backslash n_{i}=1$. Two routes $\left(n_{1}, \ldots, n_{k}\right)$, $\left(n_{1}^{\prime}, \ldots, n_{l}^{\prime}\right)$ connecting $m_{1}$ and $m_{2}$ are said to be distinct if $n_{i} \neq n_{j}^{\prime}$ except $n_{1}$ $=n_{1}^{\prime}, n_{k}=n_{l}^{\prime}$. If $m_{1} \dot{<} m_{2}$, the number of distinct routes connecting $m_{1}, m_{2}$ is denoted $v\left(m_{1}, m_{2}\right)$.

For each $m$ we have a right process $X_{t}^{m}$ on $E_{\Delta}$ with proper state space $E_{m}$ and finite proper lifetime $\zeta_{m}=T_{F_{m}}$, and an associated $\mathrm{MF} J_{t}^{m}$, as in Subsect. 4.1 .

Fix $N$ for which there exist $m \dot{<} N$. For $m_{1} \dot{<} m_{2} \leqq N\left(m_{2} \dot{<} N\right.$ or $\left.m_{2}=N\right)$, let $\Psi_{t}^{m_{2} m_{1}}$ be the MF in $X_{t}^{N}$ defined in a similar way to the MF $\Psi_{t}^{m}$ at the beginning of Subsect. 4.2, with the roles of $X_{t}^{m}, X_{t}^{m+1}$ and $U_{m}$ replaced by $X_{t}^{m_{1}}$, $X_{t}^{m_{2}}$ and $U_{m_{1}}$. That is, with $\widetilde{\Psi}_{t}^{N m}$ (resp. $\widetilde{\Psi}_{t}^{N m_{2} m_{1}}$ ) denoting the TMF $\Psi_{t}$ in Sect. 3 when $X_{t}^{N}, X_{t}^{m_{1}}$ (respectively $X_{t}^{m_{2}}$ ), $E_{N}, E_{m_{1}}$ (respectively $E_{m_{2}}$ ) and $U_{m_{1}}$ play the roles of $X_{t}^{2}, X_{t}^{1}, G_{2}, G_{1}$ and $U$ in Sect. 3, we have

$$
\Psi_{t}^{m_{2} m_{1}}=\bar{\Psi}_{t}^{N m_{1}} / \widetilde{\Psi}_{t}^{N m_{2} m_{1}}
$$

(A precise definition of $\Psi_{t}^{m_{2} m_{1}}$ is in the proof of Theorem 4.2 below.)

\section{Definition.}

$$
\hat{\Psi}_{t}^{N}=\prod_{\substack{m_{1}<m_{2} \leqq N \\ m_{2} \backslash m_{1}=1}} \Psi_{t}^{m_{2} m_{1}} \prod_{\substack{m_{1}<m_{2} \leqq N \\ m_{2} \backslash m_{1}>1}}\left(\Psi_{t}^{m_{2} m_{1}}\right)^{-v\left(m_{1}, m_{2}\right)+1} .
$$

Note that in the situation of Theorem 1, i.e. that in Subsect. 4.2, $\hat{\Psi}_{t}^{N}$ reduces to $\Psi_{t}^{N-1} \ldots \Psi_{t}^{1}$.

Theorem 4.2 (i) $\hat{\Psi}_{t}^{N}$ is a TMF in $X_{t}^{N}$.

(ii) If $N \dot{<} N^{\prime},\left.\left.X_{t}^{N^{\prime}} \cdot \hat{\Psi}_{t}^{N^{\prime}}\right|_{U_{N}} \sim X_{t}^{N} \cdot \hat{\Psi}_{t}^{N}\right|_{U_{N}}$.

Proof. The proof of (i) is similar to that of Theorem 4.1. Define, for $m_{1} \dot{<} m_{2} \leqq N$, $\phi_{t}^{m_{2} m_{1}}$ as in (4.11). Define, for $m<N, \overline{\mathscr{F}}_{t}^{N}$-stopping times $T_{n j}^{m}, R_{n j}^{m}$ as in (4.12). Then define for $m \leqq m_{1} \dot{<} m_{2} \leqq N$ the approximating functionals $\Phi_{n}^{m_{2} m_{1} m}(t)$ and $\Phi_{n}^{m_{2} m}(t)$ as in (4.13). We have a.s.

$$
\Phi_{n}^{m_{2} m_{1}}(t) \rightarrow \Psi^{m_{2} m_{1}}(t) \text { uniformly in } t, \quad \text { as } n=n_{k}\left(X_{0}^{N}\right) \rightarrow \infty
$$

where $n_{k}(x)$ is defined in the same way as in Subsect. 4.2 to guarantee a sufficient

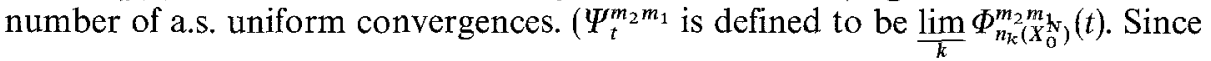

all $\Psi_{t}^{m_{2} m_{1}}$ are strictly positive $\mathrm{MFs} \hat{\Psi}_{t}^{N}$ is a well-defined MF. To show it is a TMF, it suffices to show $P_{x}^{N}\left[L_{n}(S)\right] \leqq 1$ for an $\mathscr{F}_{t}^{N}$-stopping time $S \leqq \zeta_{N}$, where

$$
L_{n}(t)=\prod_{\substack{m_{1}<m_{2} \leqq N \\ m_{2} \backslash m_{1}=1}} \Phi_{n}^{m_{2} m_{1}}(t) \cdot \prod_{\substack{m_{1}<m_{2} \leqq N \\ m_{2} \backslash m_{1}>1}}\left(\Phi_{n}^{m_{2} m_{1}}(t)\right)^{-v\left(m_{1}, m_{2}\right)+1} .
$$

Define for $m \dot{<} N$ disjoint (increasing) stochastic intervals $\left[\tau_{n j}^{m}, \gamma_{n j}^{m}\right.$ ) by the requirement

$$
\bigcup_{j}\left[\tau_{n j}^{m}, \gamma_{n j}^{m}\right)=\bigcup_{j}\left[T_{n j}^{m}, R_{n j}^{m}\right)-\bigcup_{m^{\prime}<m} \bigcup_{j}\left[T_{n j}^{m^{\prime}}, R_{n j}^{m^{\prime}}\right)
$$


(the set difference being proper), so

$$
\bigcup_{j}\left[T_{n j}^{m_{1}}, R_{n j}^{m_{1}}\right)=\bigcup_{m \leqq m_{1}} \bigcup_{j}\left[\tau_{n j}^{m}, \gamma_{n j}^{m}\right) .
$$

Now

$$
\begin{aligned}
L_{n}(t)= & \prod_{\substack{m_{1}<m_{2} \leqq N \\
m_{2} \backslash m_{1}=1}} \prod_{j} \phi^{m_{2} m_{1}}\left[T_{n j}^{m_{1}} \wedge t, R_{n j}^{m_{1}} \wedge t\right) \\
& \cdot \prod_{\substack{m_{1}<m_{2} \leqq N \\
m_{2} \backslash m_{1}>1}} \prod_{j}\left(\phi^{m_{2} m_{1}}\left[T_{n j}^{m_{1}} \wedge t, R_{n j}^{m_{1}} \wedge t\right)\right)^{-v\left(m_{1}, m_{2}\right)+1} \\
= & \prod_{m<N} \prod_{j}\left[\prod_{\substack{m \leqq m_{1}<m_{2} \leqq N \\
m_{2} \backslash m_{1}=1}} \phi^{m_{2} m_{1}}\left[\tau_{n j}^{m} \wedge t, \gamma_{n j}^{m} \wedge t\right)\right. \\
& \left.\cdot \prod_{\substack{m \leqq m_{1}<m_{2} \leqq N \\
m_{2} \backslash m_{1}>1}}\left(\phi^{m_{2} m_{1}}\left[\tau_{n j}^{m} \wedge t, \gamma_{n j}^{m} \wedge t\right)\right)^{-v\left(m_{1}, m_{2}\right)+1}\right] .
\end{aligned}
$$

The product inside the brackets simplifies to $\phi^{N m}\left[\tau_{n j}^{m} \wedge t, \gamma_{n j}^{m} \wedge t\right)$. The reason is roughly that if $m_{1} \dot{<} m_{2}$ and $v\left(m_{1}, m_{2}\right)=v$, then there exist $v$ distinct routes connecting $m_{1}, m_{2}$, and corresponding to each such route $\left(m_{1}=n_{1}, n_{2}, \ldots, n_{k}\right.$ $=m_{2}$ ) the first product inside the above brackets contains the subproduct $\left(\phi^{n_{2} n_{1}} \phi^{n_{3} n_{2}} \ldots \phi^{n_{k} n_{k-1}}\right)\left[\tau_{n j}^{m} \wedge t, \gamma_{n j}^{m} \wedge t\right)=\phi^{m_{2} m_{1}}\left[\tau_{n j}^{m} \wedge t, \gamma_{n j}^{m} \wedge t\right)$, and now its $v^{\text {th }}$ power multiplied by the corresponding factor in the second product inside the brackets is $\phi^{m_{2} m_{1}}\left[\tau_{n j}^{m} \wedge t, \gamma_{n j}^{m} \wedge t\right.$ ). Successive "cancellations" like this will finally yield the simplified expression. Therefore,

$$
L_{n}(t)=\prod_{m<N} \prod_{j} \phi^{N m}\left[\tau_{n j}^{m} \wedge t, \gamma_{n j}^{m} \wedge t\right) .
$$

Now the rest of the proof of (i) is the same as the last part (the simplification of $L_{n}(t)$ there) of the proof of Theorem 4.1. To prove (ii), we claim

$$
\hat{\Phi}_{t}^{N^{\prime}}=\phi_{t}^{N^{\prime} N} \hat{\Phi}_{t}^{N} \text { on }\left[0, T_{U_{N}^{c}}\right] .
$$

For on $\left[0, T_{U_{N}^{c}}\right]$, if $N \leqq m_{1} \dot{<} m_{2} \leqq N^{\prime}$ then $\Psi_{t}^{m_{2} m_{1}}=\phi_{t}^{m_{2} m_{1}}$; so

$$
\prod_{\substack{N \leqq m_{1} \dot{<} m_{2} \leqq N^{\prime} \\ m_{2} \backslash m_{1}=1}} \Psi_{t}^{m_{2} m_{1}} \prod_{\substack{N \leqq m_{1}<m_{2} \leqq N^{\prime} \\ m_{2} \backslash m_{1}>1}}\left(\Psi_{t}^{m_{2} m_{1}}\right)^{-v\left(m_{1}, m_{2}\right)+1}=\phi_{t}^{N^{\prime} N}
$$

by the same argument as the one used to simplify $L_{n}(t)$, and (4.16) follows from this and the definitions of $\Phi_{t}^{N}$ and $\Phi_{t}^{N^{\prime}}$. Since $\left.\left.X_{t}^{N^{\prime}} \cdot \phi_{t}^{N^{\prime} N}\right|_{U_{N}} \sim X_{t}^{N}\right|_{U_{N}}$, (ii) is established.

Define for each $N$

$$
\begin{aligned}
Y_{t}^{N} & =\left.X_{t}^{N}\right|_{U_{N}} & & \text { if there exist no } m \dot{<} N, \\
& =\left.X_{t}^{N} \cdot \hat{\Psi}_{t}^{N}\right|_{U_{N}} & & \text { if there exist } m \dot{<} N .
\end{aligned}
$$

$Y_{t}^{N}$ is a right process (actually a standard process) on $E_{A}$ with proper state space $U_{N}$ and finite proper lifetime $\zeta_{N}^{\prime}=T_{U_{N}^{c}}$. From (ii) of Theorem 4.2 the family $\left\{Y_{N}\right\}$ is consistent, in the sense that for any $N, N^{\prime}$ we have $\left.\left.Y_{N}\right|_{U_{N} \cap U_{N^{\prime}}} \sim Y_{N^{\prime}}\right|_{U_{N} \cap U_{N^{\prime}}}$. 
Again by the theorem in [8] there exists a right process (actually a standard process) $Y_{t}$ on $E_{\Delta}$ such that

$$
\left.Y_{t}\right|_{U_{N}} \sim Y_{t}^{N}, \quad N \geqq 1
$$

Define a $\mathrm{MF} M_{t}^{N}$ in $Y_{t}^{N}$ by

$$
\begin{aligned}
M_{t}^{N} & =J_{t \wedge \zeta_{N}^{N}}^{N} & & \text { if there exist no } m \dot{<} N \\
& =J_{t \wedge \zeta_{N}^{N}}^{N}\left(\hat{\Psi}_{t \wedge \zeta_{N}^{\prime}}^{N}\right)^{-1} & & \text { if there exist } m \dot{<} N .
\end{aligned}
$$

(Compare with (4.8)). The trajectory-dependent $M_{t}^{N}$ are consistent: $M_{t}^{N}=M_{t}^{N^{\prime}}$ on $\left[0, \zeta_{N}^{\prime} \cap \zeta_{N^{\prime}}^{\prime}\right]$; see (4.9). Therefore we can define a $\mathrm{MF} M_{t}$ in $Y_{t}$ is an obvious way so that $M_{t}=M_{t}^{N}$ on $\left[0, \zeta_{N}^{\prime}\right]$ for all $N$. That $\left(Y_{t}, M_{t}\right)$ is as desired is proved as at the end of Subsect. 4.1.

\section{References}

1. Bliedtner, J., Hansen, W.: Potential theory. Berlin Heidelberg New York: Springer 1986

2. Blumenthal, R.M., Getoor, R.K.: Markov processes and potential theory. New York: Academic Press 1968

3. Constantinescu, C., Cornea, A.: Potential theory in harmonic spaces. Berlin Heidelberg New York: Springer 1972

4. Getoor, R.K.: Markov processes: Ray processes and right processes. (Lect. Notes Math., vol. 440) Berlin Heidelberg New York: Springer 1975

5. Ito, K., Watanabe, S.: Transformation of Markov processes by multiplicative functionals. Ann. Inst. Fourier 15, 13-30 (1965)

6. Sharpe, M.J.: General theory of Markov processes. San Diego: Academic Press 1988

7. Shih, C.T.: Construction of right processes from hitting distributions. In: Çinlar, E. et al. (eds.) Seminar on stochastic processes, pp. 189-236. Boston Basel Stuttgart: Birkhäuser 1984

8. Shih, C.T.: On piecing together locally defined processes. In: Çinlar, E. et al. (eds.) Seminar on stochastic processes, pp. 321-333. Boston Basel Stuttgart: Birkhäuser 1991

9. Shih, C.T.: Construction of Markov processes from hitting distributions without quasi-leftcontinuity. In: Çinlar, E. et al. (eds.) Seminar on stochastic processes, pp. 187-231. Boston Basel Stuttgart: Birkhäuser 1992 